\title{
In-Situ Liquid Phase TEM Observations of Nucleation and Growth Processes
}

\author{
James J. De Yoreo ${ }^{1,2, *}$ \\ ${ }^{1}$ Physical Sciences Division, Pacific Northwest National Laboratory, Richland, WA 99352; \\ ${ }^{2}$ Department of Materials Science and Engineering and Department of Chemistry, University of \\ Washington, Seattle, WA, 98195;
}

*Correspondence should be directed to:

J.J. De Yoreo

PNNL

PO Box 999, MS K2-01

Richland, WA 99352

Phone: 509-375-6494

FAX: 509-371-6242

E-mail: james.deyoreo@pnnl.gov

\begin{abstract}
Nucleation and growth of crystals is a pervasive phenomenon in the synthesis of manmade materials, as well as mineral formation within geochemical and biological environments. Over the past two decades, numerous ex situ studies of crystallization have concluded that nucleation and growth pathways are more complex than envisioned within classical models. The recent development of in situ liquid phase TEM (LP-TEM) has led to new insights into such pathways by enabling direct, real-time observations of nucleation and growth events. Here we report results from LP-TEM studies of $\mathrm{Au}$ nanoparticle, $\mathrm{CaCO}_{3}$ and iron oxide formation. We show how these in situ data can be used to obtain direct evidence for the mechanisms underlying crystallization, as well as dynamic information that provides constraints on important kinetic and thermodynamic parameters not available through ex situ methods.
\end{abstract}

Key words: In situ TEM, nucleation, crystal growth, oriented attachment, calcium carbonate, iron oxide

\subsection{Introduction}

(C) 2016. This manuscript version is made available under the Elsevier user license http://www.elsevier.com/open-access/userlicense/1.0/ 
Nucleation and growth of inorganic crystals from solutions occurs throughout geochemical, biological, and synthetic systems. The inherent three dimensional character of these systems, combined with the immersion of the constituent building units within a solution, severely limits the range of tools that can provide molecular-to-nanoscale insights into the mechanisms and controls on these processes. Optical microscopy provides excellent time resolution and has been used to track nucleation rates, [1] but can not resolve structures below a few hundred $\mathrm{nm}$ in diameter without fluorescent labeling. X-ray scattering and spectroscopic techniques are able to detect nuclei at early times, but only capture the ensemble evolution of the system, [2] generally with long signal collection times. In situ scanning probe microscopy has been successfully applied to nucleation and growth in many surface-mediated systems due to its ability to image surfaces with high spatial resolution. [3-5] However, the early events in the formation of many materials occur rapidly relative to the time required for scanning probe image acquisition. Although recent advances in high speed scanning have greatly improved the attainable temporal resolution [6], surface scanning techniques still place severe limits on sample type and geometry. Scanning probe microscopy is of little use, for example, in examining crystallization pathways that involve aggregation or ordered assembly of molecular clusters [7] or post-nucleation particles $[8,9]$ in bulk solutions. Similarly, nucleation events that occur within organic matrices, such as globular phases of macromolecules, [10] lipid vesicles, [11, 12] amelogenin, [13] and collagen, [14] cannot be accessed via scanned probe methods.

As a consequence of these limitations, LP-TEM [15-18] has filled a critical gap in the suite of experimental tools available for investigating crystallization processes in solutions. It combines the temporal and spatial resolution of electron microscopy with the ability to image events occurring in bulk solutions. This technique has been applied to a broad range of materials systems including metallic and semiconductor nanoparticles and nanowires, [9, 18-39] geochemical and biological minerals, [8, 10, 40-42] electrochemical systems (see Ref. [17] for a recent review), protein complexes, [40, 43, 44] viruses, and self-assembling systems of organic films, vesicles, macromolecules, and nanoparticles. [10, 29, 43, 45-48] Moreover, nucleation and growth events can be triggered within LP-TEM liquid cells using a number of methods, including mixing of reagents, [41] in-diffusion of gaseous a reactant, [10] electrochemical reaction, [17, 18] heating, [49] and through the radiolytic effects of the electron beam. [50-52] Processes ranging from nanoparticle nucleation to particle growth, self-assembly, and coarsening 
have been explored in both hard and soft-matter systems. The purpose of this article is to illustrate the use of LP-TEM to gain new insights into nucleation and growth processes by reviewing four recent examples that utilize distinct methods to drive crystallization.

\subsection{Materials and Methods}

\subsection{Liquid cell designs}

LP-TEM sample holders closely resemble standard TEM holders, except that the tip is designed to support a sample cell that isolates a liquid volume from the vacuum environment of the TEM column (Fig. 1a,b). [15, 18, 37, 38, 53, 54] In the case of flow systems, tubing runs down the center of the holder to provide one or more inlets and an outlet for the liquid (Fig. 1c). $[41,42]$ Three types of fluid cells have been used for LP-TEM investigations of nucleation and growth. The first type, which is based on the original design by Ross and co-workers, is a static microfabricated cell composed of two silicon wafers with silicon nitride films coating both sides of each wafer (Fig. 1a). [18] The silicon is etched away to produce nitride membranes that constitute the electron-transparent windows. Etching through both layers creates solution access points on either side of the imaging area, to which Si towers are glued as solution reservoirs. Additionally, on the lower wafer a $10-500 \mathrm{~nm}$ silicon nitride spacer is deposited to create a fluid layer of an appropriate thickness. To perform an experiment, the two wafers are glued together, solution is introduced into the reservoirs, and these are sealed with glass caps. This basic design has been modified to enable electrochemical and thermal control using one of a number of approaches (Fig. 1b).

A second version of static liquid cells exploits the electron transparency of graphene sheets. [37] These cells are fabricated by injecting solution between two graphene sheets supported on opposing TEM grids. Strong van der Waals interactions hold the graphene sheets together, creating solution filled pockets for imaging. While the graphene provides excellent electron transparency, as with the microfabricated $\mathrm{Si}$ cells, the resolution is nonetheless determined by the thickness of the solution layer. Moreover, like the microfabricated Si cells, the only dynamic processes that can be investigated are currently limited to nanoparticle interactions through Brownian motion or processes driven by radiolysis of the liquid.

Because many synthesis processes result from mixing of reagents and/or the process can only continue as long as fresh solution is delivered to the reaction volume, designs that enable 
flow through the imaging volume have emerged as the most versatile (Fig. 1c). [20, 35] As with the original static cells, these are microfabricated Si-based devices for which vacuum isolation is achieved using O-ring seals. While most designs allow for a flow of a single solution stream to the tip, others incorporate two flow lines for reagent mixing. [10, 41] The cells have a liquid flow pathway defined by the pattern of the spacer layer, which extends across the entirety of the cell between the openings that serve as inlets and outlets. The cells are sealed from the microscope's vacuum by o-rings compressed between the stage and the cell. Liquid is pumped through one or multiple inlets in the stage, depending on design. In the case of dual-inlet systems, the two streams merge near the entrance to the liquid cell. Most of this reagent stream flows around the outside of the cell in the chamber in which the cell is placed, with an outlet tube carrying the effluent to a collection container. A small shunt from this main flow passes into the cell and through the flow channel. This split-flow geometry is necessary, because the pressure that would be required to drive the entire reagent stream through the cell would be tremendous and would rupture the cell's electron transparent membranes.

While static cells often require an external driving force to initiate the reaction, they have an advantage in the well-defined starting solution conditions within the cells. By contrast, the flow patterns within, and mixing characteristics of, flow cells and holders are largely unknown, leading to a degree of uncertainty as to the solution composition in the imaging area, particularly in the case of multiple inlet flow holders. These latter systems do, however, provide the capability of mixing multiple reagents to create supersaturated solutions and drive nucleation and growth processes in the absence of external stimuli. Furthermore, they can be used to passively diffuse gas into a liquid initially injected into the fluid cell, [10] broadening the experimental approaches feasible with such devices.

\subsection{Imaging modes and beam effects}

Imaging in both TEM- and STEM-mode has proven valuable and each has its advantages. $[15,17]$ STEM generally provides higher resolution when the cell thickness is greater than about $50 \mathrm{~nm}$ (Fig. 2). However, below that thickness, TEM imaging can provide better resolution. In addition, because the beam is rastered in STEM-mode, it generally leads to lower electron doses, but the use of short exposure times and large spot sizes during TEM imaging can be used to 
obtain electron doses rivaling those utilized even in cryogenic TEM (cryo-TEM) studies on soft matter. Beyond imaging, important analyses available in LP-TEM include electron diffraction for determination of structure, [41] energy filtered imaging for elemental differentiation, [49] electron energy loss [55] and energy dispersive [34] spectroscopies for compositional analysis.

The solution composition in the fluid cell can be strongly affected by the electron beam, which produces many radiolysis products that can directly impact nucleation and growth processes. [56] For example, hydrated electrons created by the interaction of the beam with water lead to metal ion reduction, which greatly accelerates the growth of metallic particles. However, even with non-metallic materials, the interaction of the electron beam with the solution can alter nucleation and growth processes through indirect effects including shifts in $\mathrm{pH}$ due to production of $\mathrm{H}^{+}$or shifts in solution composition though more complex radiolysis reactions, all of which can impact the supersaturation, reaction kinetics, and interaction potentials experienced by particles and solution species. Impacts on, for example, nucleation rates are will documented. [35] Consequently, care must be taken to demonstrate that the observed process is representative of the system in the absence of the beam by performing measurements as a function of electron dose, carrying out the same experiment for a period of time before exposure to the beam, and examining previously unexposed regions far enough from the zone of exposure that the resulting chemical gradients have decayed away. [10,41]

\subsection{Results}

\subsection{Au nanoparticle nucleation: an illustration of classic nucleation behavior}

According to classical nucleation theory (CNT), density fluctuations give rise to incipient nuclei. [57-59] In a supersaturated regime, the negative change in chemical potential associated with precipitation provides a driving force for spontaneous growth of these incipient nuclei that scales with the number of growth units that transition to the solid state, giving a term that is cubic in the radius of the nucleus. However, these nuclei also possess a finite interfacial free energy, leading to a positive term in the free energy change that scales with the surface area and is thus is quadratic in the radius. At very small size, the quadratic term exceeds the cubic term. Consequently, these incipient nuclei can only grow by increasing the free energy until the radius exceeds the critical value, $r_{c}$. Thus, below this size, particles will, on average, shrink rather than 
grow, but beyond this size, the free energy then decreases with further growth and hence, on average, particles will grow rather than shrink.

A simple LP-TEM experiment was used to look at the dynamics of nucleation and search for evidence of classical behavior. [60] Static microfabricated silicon nitride fluid cells (Fig. 1a,b) were used to observe the dynamics of gold nanoparticle nucleation triggered by exposure to the electron beam. Solutions of gold chloride made by mixing $0.5 \mathrm{~mL}$ of a $0.01 \mathrm{M} \mathrm{Na}$-citrate solution and $0.5 \mathrm{~mL}$ of $0.01 \mathrm{M} \mathrm{HAuCl}_{4}$ solution with $18 \mathrm{~mL}$ of DI water were added to freshly prepared $\mathrm{NaBH}_{4}$ solution $(0.3 \mathrm{~mL}$ of $0.01 \mathrm{M})$, which acted as a reductant to form the gold nanoparticles. The solutions were stored at room temperature for a period of less than a month prior to injection into the fluid cells.

Upon exposure to the electron beam, existing Au nanoparticles began to grow while many new particles formed over the course of the experiment (Fig. 3). While numerous successful particle formation events were observed, there were many instances in which a $\mathrm{Au}$ particle began to form, fluctuated in size, and eventually dissolved away rather than growing into a stable crystal, despite the fact that many particles of similar size succeeded in achieving stable growth.

This behavior is consistent with the expectations of classical nucleation theory.

Estimating the critical radius from the maximum diameter reached by incipient nuclei that dissolve instead of continuing to grow, the TEM experiments gave a critical radius of $25 \mathrm{~nm} \pm 4$ $\mathrm{nm}$ (based on measurements of eight particles where the error is one std. dev.) Unfortunately, because neither the value of the interfacial free energy for citrate-capped Au nor the supersaturations of the solution under the beam are known, the value of $r_{c}$ can not be used to estimate either quantity independently and thus check whether the observed behavior is in quantitative agreement with the predictions of CNT.

\subsection{Nucleation via multiple direct and indirect pathways}

Ex situ observations of precipitates in systems like $\mathrm{CaCO}_{3}$ that exhibit numerous polymorphs are often cited as evidence for the Oswald-Lussac rule of stages, [61-63] which holds that the polymorphs of a crystal will appear successively during nucleation in the order of the least to most stable. To investigate the order and dynamics of polymorph nucleation, a dual inlet LP-TEM stage (Fig. 1c) was used to observe formation of $\mathrm{CaCO}_{3}$ precipitated by mixing 
two precursor salt solutions to create a supersaturated solution in the imaging area. [41] $\mathrm{CaCl}_{2}$ and $\mathrm{NaHCO}_{3}$ solutions of varying concentration were introduced into the flow cell using a range of flow rates set independently for the two reagents. In some experiments, supersaturations increased initially, but eventually decreased until undersaturated so that dissolution was also observed as a single reagent continued to flow into the cell. Calculated supersaturations for the various phases ranged from undersaturated to highly supersaturated, assuming perfect mixing of the reagent streams. All images were collected in TEM mode. However, the microscope was periodically switched to diffraction mode in order to determine the phase of the forming solids.

Nucleation of both metastable and stable phases, including amorphous calcium carbonate (ACC), vaterite, aragonite, and calcite, was observed. The precipitates exhibited morphologies common for these phases when observed ex situ. Increasing the initial ion concentrations generally resulted in an increase in the number of $\mathrm{CaCO}_{3}$ phases observed. In fact, on many occasions multiple phases were observed to occur during a single experiment. Moreover, in a number of experiments initially formed metastable phases transformed into more stable phases to produce indirect pathways of nucleation.

The sequences in Fig. 4 and Fig. 5 illustrate formation of all four calcium carbonate phases through direct or indirect pathways. Figure 4 shows the simultaneous, direct formation of the stable calcite phase and metastable vaterite. Two calcite rhombohedra formed in the absence of any visible precursor and grew throughout the following minute. Concomitantly, a particle with ellipsoidal projection formed and grew into contact with the central rhomb. Obvious diffraction contrast was not observed nor was a diffraction pattern acquired, so there is some ambiguity as to the identity of this particle's phase as both ACC and vaterite have been observed to have similar projections. However, in other experiments where either ACC or vaterite formation were confirmed by diffraction, ACC was found to be circular in projection while vaterite typically exhibited an ellipsoidal geometry. Moreover, prior studies found that ACC dissolves in the presence of calcite $[1,64]$ and, as shown in the following example, is rapidly consumed when in contact with crystalline phases. In this first example, the two phases grew concurrently after coming into contact, again indicating the second phase was vaterite rather than ACC.

Figure 5 shows the formation and growth of a single ACC particle. The amorphous particle grew to a few microns in diameter, before briefly shrinking in size prior to nucleation of 
two particles at or just below the surface of the ACC precursor, both of which exhibited the typical sheath-of-wheat morphology of aragonite. [65] The aragonite grew rapidly, consuming the amorphous precursor, with physical contact maintained between the two phases throughout the transformation process. In each observation of similar multi-step pathways, the crystals appeared to form through heterogeneous nucleation on or just below the surface of the precursor particle, rather than through a physically separated nucleation event followed by dissolution of the more soluble ACC phase.

Figure 6 provides a summary of the various formation pathways observed in the study of Nielsen et al. [41] Observed pathways included direct formation of all three anhydrous crystalline phases, as well as ACC, while multi-step pathways led to formation of both aragonite and vaterite via ACC. Interestingly, despite performing more than one hundred experiments, formation of calcite via ACC was never observed, indicating that the barrier to nucleation from ACC is significantly higher than for either of the other two anhydrous crystalline phases. As Fig. 6 indicates, in at least one instance, calcite appeared to form on and grow at the expense of an initially formed aragonite crystal. However, given that TEM images are 2D projections of 3D volumes, there is some uncertainty as to whether the two crystals were initially in contact.

Measurements of growth rates for the various phases indicated that, despite uncertainties about the extent of reagent mixing and the rate of flow through the imaging area itself, lateral growth rates following nucleation exhibited linear growth curves (Fig. 7). This linear dependence implies that growth was limited by surface reaction kinetics rather than bulk diffusion. [66] However, while a single growth rate described the entire growth period for some experiments, in others two distinct rates were observed (See for example, Fig. 7a and the inset to Fig. 7b). In all of the latter cases the initial rate was always greater than the second. This decrease in growth rate indicates a drop in suprsaturation and may have been the result of nucleation events upstream of the imaging area, which reduced the supersaturation or partially blocked reagent flow into the cell. These results demonstrate that quantitative analysis of rates independent of limitations by diffusive transport are possible with LP-TEM flow cells.

\subsection{Control over nucleation by an organic matrix}

In many biomineralizing systems, the formation of mineral components often occurs within a hydrated macromolecular matrix that is rich in biopolymers with numerous charged side 
groups [67, 68]. Based on in vitro evidence, these molecules are assumed to play a role in stabilizing amorphous precursor phases from which many crystalline biominerals are formed. In particular, immobilized, highly charged macromolecules, such as polysaccharides, have been proposed to act as concentrators of mineral ions by cation binding prior to crystallization [69, $70]$.

The importance of ion binding in promoting nucleation was recently investigated by Smeets et al. [10], who used LP-TEM to examine the effects of polystyrene sulfonate (PSS) on $\mathrm{CaCO}_{3}$ nucleation. With an abundance of sulfonate groups, the PSS served as a surrogate for highly charged biomolecular matrices. To conduct this study, control experiments diffused $\mathrm{CO}_{2}$ into $\mathrm{CaCl}_{2}$ solutions within silicon nitride cells in the absence of PSS. These experiments resulted in the nucleation and growth of vaterite, which formed randomly throughout the experimental volume. In contrast, when PSS was introduced to the $\mathrm{CaCl}_{2}$ solution prior to introducing $\mathrm{CO}_{2}$, the $\mathrm{Ca}^{2+}$ complexed with the PSS to form Ca-PSS globules. These were approximately 10-20 $\mathrm{nm}$ in diameter and were evident in TEM, AFM and dynamic light scattering measurements (Fig. 8a,b). FTIR and zeta potential data demonstrated that the $\mathrm{Ca}^{2+}$ interacted with the negatively charged sulfonate groups (Fig. 8c,d) and titration calorimetry showed that $56 \%$ of the $\mathrm{Ca}^{2+}$ in the solution was complexed within the globules.

Further experiments found that when $\mathrm{CO}_{2}$ was diffused into the TEM fluid cells containing the globules, the first phase to appear was ACC rather than vaterite [10]. Moreover, the ACC nanoparticles nucleated exclusively within the globules (Fig. 9). At much later times, after growth of the ACC particles had ceased, vaterite formed sporadically in regions away from the globules. Analysis of the ACC and vaterite growth rates (Fig. 10) showed that ACC nucleation in the presence of PSS occurred at a higher supersaturation $(\sigma=1.0-1.8$ relative to $A C C)$ than the nucleation of vaterite in the absence of PSS $(\sigma=0.5-0.6$ relative to vaterite). Because ACC has a lower surface energy [71, 72], ACC nucleation is expected to be more likely than vaterite nucleation at equal supersaturation. Consequently, these results show that the addition of PSS significantly inhibits ACC nucleation. The implication is that although PSS controls the location of nucleation and stabilizes the amorphous phase, it does not act by lowering the interfacial free energy, which would instead lead to a reduction in the supersaturation needed for nucleation. Instead, these results show that the negatively charged polymer controls nucleation by binding calcium ions to create a high $\mathrm{Ca}^{2+}$ concentration within 
the globules and a low concentration everywhere else. The localized formation of the metastable ACC then results from both the locally high supersaturation and interaction with the stabilizing polymer matrix, while the reduced calcium concentration in solution prevents immediate vaterite crystallization outside the globules. Thus the results demonstrate the significant role that ion binding can play in directing nucleation.

\subsection{Growth by particle aggregation and oriented attachment}

Following nucleation of nanoparticles, their interaction can lead to growth through either coarsening or aggregation and coalescence. LP-TEM provides a unique and direct method for investigating these processes, as well as for determining whether particles become crystallographically aligned during coalescence events. This latter process is often referred as oriented attachment (OA). Generally speaking, the resolution available in flow cells, which use an O-ring system to seal the chamber, is insufficient to perform such experiments, because the resolution is too low to determine the crystallographic orientations of particles, (unless they happen to have an extreme aspect ratio that is uniquely correlated with crystallographic orientation [50]). However, static cells, using both Si-based microfabricated chips and graphene films, have enabled lattice resolution in a number of studies. [8, 9, 27] With silicon-based cells, there are two potentials ways to achieve this resolution. The first and most useful results from inward bowing of the thin electron-transparent windows so that the membrane separation is below about $100 \mathrm{~nm}$. However, bowing is random and, for many cells, the windows either end up touching so that there is no fluid in the imaging volume or they bow outward so that the separation is over one micron and the resolution is poor. The second way to achieve lattice resolution is to image with high electron beam current in a single location. Radiolysis effects will often cause movement of the fluid from the imaging region leaving a thin meniscus that progressively thickens as one moves away from this region. [42]

Li et al. $[8,60]$ used static Si-based liquid cells, in which the bowing resulted in a thin liquid layer through much of the cell, to observe aggregation and coalescence of ferrihydrite (iron oxyhydroxide) nanoparticles at lattice resolution. Iron oxide particle synthesis was achieved by mixing $\mathrm{FeCl}_{3}(20 \mathrm{mM})$ and $\mathrm{KH}_{2} \mathrm{PO}_{4}(0.45 \mathrm{mM})$ solutions with a $\mathrm{pH}$ of 2.05 to 2.08 . A few microliters of the $\mathrm{FeCl}_{3}-\mathrm{KH}_{2} \mathrm{PO}_{4}$ solution was loaded into the liquid cell, which was sealed and placed into the microscope. Rod-like akaganeite nanoparticles produced in ex situ 
reactions with this solution at $100-150{ }^{\circ} \mathrm{C}$ for $0.5-48 \mathrm{~h}$ subsequently transformed into other iron oxide phases (23). Selected Area Electron Diffraction pattern analysis indicates that the nanoparticles formed in the fluid cell are closely related to six-line ferrihydrite $\left(5 \mathrm{Fe}_{2} \mathrm{O}_{3} \cdot 9 \mathrm{H}_{2} \mathrm{O}\right)$. Consequently, the chain of events is as follows: akaganeite serves as a precursor to the ferrihydrite phase. That phase forms from the akaganeite through a classic dissolutionreprecipitation mechanism.

Low-resolution TEM images showed that the ferrihydrite nanoparticles underwent aggregation and coalescence (Fig. 11). High-resolution movies that revealed the dynamics of the attachment process: the particles continuously diffused and rotated (Fig.11a-c), repeatedly contacting one another until attachment finally occurred (Fig.11d,e). The surfaces of two adjoining particles made transient contact at many points and orientations before finally attaching through a sudden "jump-to-contact". Similar behavior was observed for smaller, 5-10 nm particles typical of 6 line ferrihydrite (23).

Lattice fringe images revealed that, irrespective of how many times particles made contact, at the time of attachment they either shared the same crystallographic orientation or their orientations were twin-related (Fig. 12a,b). Either way, following attachment, atoms filled the interface region on a timescale of order 10-100s, beginning at the point of contact. Interface elimination resulted either in larger defect-free crystals (Fig. 12a) or crystals that contained a (101) twin plane (Fig. 12b).

Analysis of particle motion leading to attachment shows that the translational and rotational speeds increased by factors of $2-4$ once the particles were within $5-10 \AA$ of one another (Fig. 13a). Because the expected Debye length for the $50 \mathrm{mM} 3: 1$ electrolyte solutions used in the experiments is also of this order.[73] Li et al.[8] proposed that the jump-to-contact is driven by electrostatic interactions. Analyzing the translational and rotational accelerations, they extracted the attractive forces and torques driving attachment and, from the distance over which acceleration occurred, calculated the potential energy of attraction. Equating this potential to a Coulombic attraction, they concluded that the particles interact with an effective number of fundamental charges (1.6E-19 C) of order unity.

Following attachment, the new crystal-solution boundary advanced away from the contact point through monomer addition, with the lateral growth rate decreasing rapidly within the first two seconds, and reaching a constant value after approximately 20 seconds (Fig. 13b). 
The dependence of the speed of the growth front on the curvature of the interface is consistent with the well-known exponential dependence of chemical potential on interface curvature (29).

Even in cases where attachment resulted in a defect-free interface with no remaining evidence that an OA event had taken place, the crystals were often slightly misaligned at the time of attachment. In the example shown in Fig. 14, the lattice planes near the boundary are slightly bent due to the formation of two edge dislocations during the attachment event. None-the-less, within two seconds, these dislocations translated laterally across the interface (Fig. 14c), leaving behind perfectly flat lattice planes and eliminating any trace of the inter-particle boundary (Fig. 14d).

Sometimes, these misalignments were sufficiently large that they led to visible postattachment particle rotation. [8, 60] The sequence in Fig. 15 shows an oblong particle presumably formed during an earlier OA event drifting by a larger branched particle formed through numerous aggregation events. As the lower left corner of the smaller particle passes near the larger mass with a relative orientation that is close to alignment, it experiences the attractive force that drives $\mathrm{OA}$ in this system. This force exerts a torque on the particle rotating it clockwise into co-alignment and causing the final jump-to-contact. However, even after the initial attachment, line defects can be seen in the interface region and their elimination leads to a visible counter-clockwise rotation.

The torque that must be applied through relaxation of the interface in order to rotate the particle in Fig. 15 through the observed post-attachment rotation angle was estimated from the dependence of the relative angle on time (Fig. 16). During the first two seconds, the particle rotated counterclockwise at a constant rate, thus the net torque was zero. This means the torque due to relaxation of the interface was approximately equal to the resistive torque due to rotation in the viscous medium. By calculating the resistive torque for this system, Nielsen et al. were again able to estimate the potential energy $\mathrm{U}$ and, from $\mathrm{U} \sim(1 / 2) \mathrm{VE} \varepsilon^{2}$ where $\mathrm{V}$ is the volume of deformation and $\mathrm{E}$ is the elastic modulus, obtained a strain $\varepsilon$ required to produce the observed rotation of about $\sim 0.01$ or $1 \%$.

Observations in the calcium carbonate system suggest a very different aggregation process than in the ferrihydrite system. [60] Here, particle fusion was also observed, but the primary particles, which were of order $5 \mathrm{~nm}$ or less and spherical, appeared to be amorphous (Fig. 17). This conclusion would be consistent with molecular dynamics simulations, [74, 75] 
which found that amorphous calcium carbonate (ACC) should be the stable phase in the nmrange, despite the fact that all macroscopic crystalline phases of $\mathrm{CaCO}_{3}$ have much lower solubility (i.e., higher stability). These primary particles interacted with and periodically attached to a larger particle created through prior aggregation events, which was crystalline as is evident from the faceted shape, the presence of lattice fringes, and the constantly changing scattering contrast. After attachment, lattice fringes can be seen in the smaller particles as well, indicating that growth in this system does not occur through OA, but rather via attachment of amorphous particles that then crystalize using the parent crystal as a template.

\subsection{Nanoparticle coarsening}

The same system described above to investigate ferrihydrite coalescence events was used to observe Ostwald-type ripening, [60] which should occur when if the local curvature of the two interacting particles differs significantly. [76] In Fig. 18, a small particle with high positive curvature approaches a large ferrihydrite surface with negative curvature. Before an attachment event can occur, the small particle dissolves and is incorporated into the larger particle. The dependence of particle size on time during the ripening process for the particle shown in Fig. 18, as well as one other not shown here is given in Fig. 19. Although the two particles appear in similar local particle-particle interaction environments, the dissolution kinetics are quite

different. For particle 1, the fit to the $3^{\text {rd }}$ power law in Fig. 19 shows that its dissolution can be described well by Ostwald ripening controlled by the volume diffusion of ionic species in a solution. [77] On the other hand, the data for particle 2 cannot be described by any power law, suggesting factors other than size, such as the relative orientations of the dissolving and growing particles or the local environment (e.g., interface curvature), play a role in the dissolution and reprecipitation rate. The accelerated dissolution rate of this particle at $>\sim 12$ s may also reflect the rapid increase in Coulombic interactions at close proximity as the effect of surface charge screening diminishes. [78]

\subsection{Discussion and Conclusion}

Classical nucleation theory (CNT) first developed by Gibbs [57] and the terrace-ledgekink model put forth by Burton Cabrera and Frank (BCF) [79] have long been widely accepted as accurate descriptions of nucleation and growth, respectively. According to CNT, [57-59] 
nucleation occurs through density fluctuations that give rise to unstable clusters, which occasionally overcome a free energy barrier through ion-by-ion addition to produce a stable seed crystal. Within this classical picture, when an electrolyte solution is undersaturated all clusters are unstable relative to the free ions, and cluster concentration falls off exponentially with size. Moreover, although the formation of metastable phases may be kinetically favorable, the thermodynamically stable phases of both the nucleus and bulk crystal are assumed to be the same.

In recent years, a number of investigations have suggested the initial formation of crystal phases follows non-classical pathways. [61] Some studies have reported the existence of multiion complexes of order $1 \mathrm{~nm}$ or more in diameter that are present prior to nucleation [80-84] and, at least in some cases, are believed to be stable constituents of the solution even in an undersaturated solution. [81, 82, 85] Birth of the solid phase in these systems then occurs through aggregation of these pre-nucleation clusters to form nanoparticles of a stable precursor phase that is often poorly ordered or amorphous and later transforms into the crystalline phase. The barrier to nucleation via this route is proposed to be minor and thus it circumvents the classical nucleation process. Still other studies, both experimental and computational, have suggested that formation of the first precipitate in the $\mathrm{CaCO}_{3}$ system results from spinodal decomposition of the solution into two liquid phases, one of which is a solute-rich dense liquid phase [86-88]. The solid phase then nucleates within this dense liquid phase.

Once nucleation is completed, according to BCF theory [79], post-nucleation growth of crystals proceeds through ion-by-ion addition to kink sites at step edges produced either by screw dislocations or, at sufficiently high supersaturation, by nucleation of two-dimensional islands. However, a number of investigations have concluded that the non-classical growth mechanism of aggregation and coalescence of nanoparticles is a common phenomenon in biomineral [89-91], biomimetic, [92-94] environmental, [94-96] and synthetic systems. [8, 60, 97-105] The resulting crystals can exhibit complex morphologies such as quasi-one dimensional chains, highly-branched nanowires, and 3D hierarchical and self-similar superstructures.[106] Despite these complexities in growth process and morphology, the resulting crystals generally exhibit single crystal diffraction patterns. [94, 107] Thus either the primary particles become co-aligned during the attachment process $[8,95,98,100,101]$ or recrystallization via dislocation and grainboundary migration occurs after coalescence. [60, 105, 108] 
Ex situ TEM data, sometimes combined with measurements of bulk solution chemistry via and particle size distributions, have traditionally provided the primary techniques for investigating these processes. $[80,100,102]$ As the examples presented above illustrate, LPTEM now enables direct observation of both the nucleation and post-nucleation evolution of nanoparticles. Because this method provides dynamic information, the structural pathways and kinetics of these processes can both be accessed as a function of solution conditions, leading to new insights into the underlying mechanism and determination of the controlling energetic parameters.

Results on Au nucleation provide direct visualization of the fluctuating nature of particle formation predicted by $\mathrm{CNT}$. Data on $\mathrm{CaCO}_{3}$ nucleation collected over a wide range of solution conditions reveals the existence of both direct and indirect nucleation pathways including singlestep nucleation of multiple polymorphs and multi-step mineral formation marked by direct transformation of initial amorphous precursor particles into crystalline phases. High resolution imaging of iron oxide and calcium carbonate nanoparticles has provided clear evidence for particle-based growth via $\mathrm{OA}$, as well as random aggregation of amorphous nanoparticles onto secondary crystals. The observation of Ca-PSS globule formation and subsequent nucleation of ACC only within the globules under conditions that otherwise lead to vaterite formation has demonstrated the utility of liquid cell TEM in observing both soft macromolecular matrices and hard mineral constituents, revealing the role of ion binding within macromolecular matrices in controlling nucleation and the impact of macromolecules on the rate of nucleation and stabilization of metastable phases. Most importantly, the continual structural and morphological evolution observed throughout the crystallization process in all three of these examples brings into relief the reality that one cannot know what process led to crystal formation simply by looking at a final morphology or phase.

The findings reported here, as well as the many recent studies carried out by others, demonstrate that liquid cell TEM has become an invaluable tool in understanding how crystals form. Continued development of the technique will broaden its utility to enable investigations of an increasing number of crystal systems and phenomena. Of particular interest would be the ability to run experiments in the liquid cell across a range of temperatures and pressures. Furthermore, in situ diagnostic measurements of solution properties such as $\mathrm{pH}$ and composition will be critical in many quantitative studies of nucleation and growth. As these capabilities 
become widely available, crystal growth scientists will begin to address the many unanswered questions concerning both classical and non-classical pathways of crystallization.

\section{Acknowledgements}

Production of this review was supported by the Laboratory Directed Research and Development Initiative on Materials Synthesis and Simulation Across Scales at the Pacific Northwest National Laboratory (PNNL). PNNL is operated by Battelle for the U.S. Department of energy under Contract DE-AC05-76RL01830.

\section{References}

1. $\mathrm{Hu}, \mathrm{Q}$. , et al., The thermodynamics of calcite nucleation at organic interfaces: Classical vs. non-classical pathways. Faraday Discussions, 2012. 159: p. 509-523.

2. Bots, P., et al., Mechanistic Insights into the Crystallization of Amorphous Calcium Carbonate (ACC). Crystal Growth \& Design, 2012. 12(7): p. 3806-3814.

3. Chung, S., et al., Self-catalyzed growth of S layers via an amorphous-to-crystalline transition limited by folding kinetics. Proceedings of the National Academy of Sciences of the United States of America, 2010. 107(38): p. 16536-16541.

4. Petsev, D.N., et al., Diffusion-limited kinetics of the solution-solid phase transition of molecular substances. Proceedings of the National Academy of Sciences of the United States of America, 2003. 100(3): p. 792-796.

5. Sleutel, M. and A.E.S. Van Driessche, Role of clusters in nonclassical nucleation and growth of protein crystals. Proceedings of the National Academy of Sciences of the United States of America, 2014. 111(5): p. E546-E553.

6. Ando, T., et al., A high-speed atomic force microscope for studying biological macromolecules. Proceedings of the National Academy of Sciences of the United States of America, 2001. 98(22): p. 12468-12472.

7. Kumar, S., et al., A Structural Resolution Cryo-TEM Study of the Early Stages of MFI Growth. Journal of the American Chemical Society, 2008. 130(51): p. 17284-+.

8. Li, D.S., et al., Direction-specific interactions control crystal growth by oriented attachment. Science, 2012. 336(6084): p. 1014-1018.

9. Liao, H.G., et al., Real-time imaging of $\mathrm{Pt}_{3} \mathrm{Fe}$ nanorod growth in solution. Science, 2012. 336(6084): p. 1011-1014.

10. Smeets, P.J.M., et al., Calcium carbonate nucleation driven by ion binding in a biomimetic matrix revealed by in situ electron microscopy. Nature Materials, 2015. 14(4): p. 394-399.

11. Tester, C.C., et al., Synthesis and stabilization of amorphous calcium carbonate within liposomes. Abstracts of Papers of the American Chemical Society, 2011. 242.

12. Tester, C.C., M.L. Whittaker, and D. Joester, Controlling nucleation in giant liposomes. Chemical Communications, 2014. 50(42): p. 5619-5622. 
13. Fang, P.A., et al., Hierarchical self-assembly of amelogenin and the regulation of biomineralization at the nanoscale. Proceedings of the National Academy of Sciences of the United States of America, 2011. 108(34): p. 14097-14102.

14. Nudelman, F., et al., The role of collagen in bone apatite formation in the presence of hydroxyapatite nucleation inhibitors. Nature Materials, 2010. 9(12): p. 1004-1009.

15. de Jonge, N. and F.M. Ross, Electron microscopy of specimens in liquid. Nature Nanotechnology, 2011. 6(11): p. 695-704.

16. Radisic, A., et al., Quantifying electrochemical nucleation and growth of nanoscale clusters using real-time kinetic data. Nano Letters, 2006. 6(2): p. 238-242.

17. Wang, C.M., H.G. Liao, and F.M. Ross, Observation of materials processes in liquids by electron microscopy. Mrs Bulletin, 2015. 40(1): p. 46-52.

18. Williamson, M.J., et al., Dynamic microscopy of nanoscale cluster growth at the solidliquid interface. Nature Materials, 2003. 2(8): p. 532-536.

19. Chen, Q., et al., 3D Motion of DNA-Au Nanoconjugates in Graphene Liquid Cell Electron Microscopy. Nano Letters, 2013. 13(9): p. 4556-4561.

20. Grogan, J.M., L. Rotkina, and H.H. Bau, In situ liquid-cell electron microscopy of colloid aggregation and growth dynamics. Physical Review E, 2011. 83(6).

21. Jungjohann, K.L., et al., In Situ Liquid Cell Electron Microscopy of the Solution Growth of Au-Pd Core-Shell Nanostructures. Nano Letters, 2013. 13(6): p. 2964-2970.

22. Jungjohann, K.L., et al., Atomic-Scale Imaging and Spectroscopy for In Situ Liquid Scanning Transmission Electron Microscopy. Microscopy and Microanalysis, 2012. 18(03): p. 621-627.

23. Kraus, T. and N. de Jonge, Dendritic Gold Nanowire Growth Observed in Liquid with Transmission Electron Microscopy. Langmuir, 2013. 29(26): p. 8427-8432.

24. Liao, H.G., K.Y. Niu, and H.M. Zheng, Observation of growth of metal nanoparticles. Chemical Communications, 2013. 49(100): p. 11720-11727.

25. Liao, H.G., et al., TEM study of fivefold twined gold nanocrystal formation mechanism. Materials Letters, 2014. 116: p. 299-303.

26. Liao, H.G. and H.M. Zheng, Liquid Cell Transmission Electron Microscopy Study of Platinum Iron Nanocrystal Growth and Shape Evolution. Journal of the American Chemical Society, 2013. 135(13): p. 5038-5043.

27. Liao, H.G., et al., Facet development during platinum nanocube growth. Science, 2014. 345(6199): p. 916-919.

28. Liu, Y.Z., et al., In Situ Visualization of Self-Assembly of Charged Gold Nanoparticles. Journal of the American Chemical Society, 2013. 135(10): p. 3764-3767.

29. Parent, L.R., et al., Direct in Situ Observation of Nanoparticle Synthesis in a Liquid Crystal Surfactant Template. Acs Nano, 2012. 6(4): p. 3589-3596.

30. Park, J., et al., 3D structure of individual nanocrystals in solution by electron microscopy. Science, 2015. 349(6245): p. 290-295.

31. Park, J., et al., Direct Observation of Nanoparticle Superlattice Formation by Using Liquid Cell Transmission Electron Microscopy. Acs Nano, 2012. 6(3): p. 2078-2085.

32. Park, J.H., et al., Control of Electron Beam-Induced Au Nanocrystal Growth Kinetics through Solution Chemistry. Nano Letters, 2015. 15(8): p. 5314-5320.

33. Sun, M.H., et al., Structural and Morphological Evolution of Lead Dendrites during Electrochemical Migration. Scientific Reports, 2013. 3. 
34. Sutter, E., et al., In situ liquid-cell electron microscopy of silver-palladium galvanic replacement reactions on silver nanoparticles. Nature Communications, 2014. 5.

35. Woehl, T.J., et al., Direct in Situ Determination of the Mechanisms Controlling Nanoparticle Nucleation and Growth. Acs Nano, 2012. 6(10): p. 8599-8610.

36. Woehl, T.J., et al., Direct Observation of Aggregative Nanoparticle Growth: Kinetic Modeling of the Size Distribution and Growth Rate. Nano Letters, 2014. 14(1): p. 373378.

37. Yuk, J.M., et al., High-resolution EM of colloidal nanocrystal growth using graphene liquid cells. Science, 2012. 336(6077): p. 61-64.

38. Zheng, H.M., et al., Observation of Single Colloidal Platinum Nanocrystal Growth Trajectories. Science, 2009. 324(5932): p. 1309-1312.

39. Zhu, G.M., et al., In situ study of the growth of two-dimensional palladium dendritic nanostructures using liquid-cell electron microscopy. Chemical Communications, 2014. 50(67): p. 9447-9450.

40. Kashyap, S., et al., Nucleation of Iron Oxide Nanoparticles Mediated by Mms6 Protein in Situ. Acs Nano, 2014. 8(9): p. 9097-9106.

41. Nielsen, M.H., S. Aloni, and J.J. De Yoreo, In situ TEM imaging of $\mathrm{CaCO}_{3}$ nucleation reveals coexistence of direct and indirect pathways. Science, 2014. 345(6201): p. 1158-1162.

42. Nielsen, M.H.D.Y., J. J., Liquid Phase TEM Investigations of Crystal Nucleation, Growth and Transformation, in New Perspectives on Mineral Nucleation and Growth: From Solution Precursors to Solid Materials, A.E.S.K. van Driessche, M.; Benning, L.G; Gebauer, D, Editor. 2016, Springer.

43. Evans, J.E., et al., Visualizing macromolecular complexes with in situ liquid scanning transmission electron microscopy. Micron, 2012. 43(11): p. 1085-1090.

44. Kashyap, S., et al., Visualization of Iron-Binding Micelles in Acidic Recombinant Biomineralization Protein, MamC. Journal of Nanomaterials, 2014.

45. Gilmore, B.L., et al., Visualizing viral assemblies in a nanoscale biosphere. Lab on a Chip, 2013. 13(2): p. 216-219.

46. Liu, L.L., et al., Visualization of film-forming polymer particles with a liquid cell technique in a transmission electron microscope. Analyst, 2015. 140(18): p. 63306334.

47. Plamper, F.A., et al., Spontaneous Assembly of Miktoarm Stars into Vesicular Interpolyelectrolyte Complexes. Macromolecular Rapid Communications, 2013. 34(10): p. 855-860.

48. Proetto, M.T., et al., Dynamics of Soft Nanomaterials Captured by Transmission Electron Microscopy in Liquid Water. Journal of the American Chemical Society, 2014. 136(4): p. 1162-1165.

49. Xin, H.L.L. and H.M. Zheng, In Situ Observation of Oscillatory Growth of Bismuth Nanoparticles. Nano Letters, 2012. 12(3): p. 1470-1474.

50. Chen, Q.C., H.; Manthiram, K.; Yoshida, M.; Ye, X.; Alivisatos, A.P., Interaction Potentials of Anisotropic Nanocrystals from the Trajectory Sampling of Particle Motion using in Situ Liquid Phase Transmission Electron Microscopy. ACS Central Science, 2015. 1: p. 33-39.

51. de Put, M.W.P.V., et al., Writing Silica Structures in Liquid with Scanning Transmission Electron Microscopy. Small, 2015. 11(5): p. 585-590. 
52. den Heijer, M., et al., Patterned electrochemical deposition of copper using an electron beam. Apl Materials, 2014. 2(2).

53. Grogan, J.M. and H.H. Bau, The Nanoaquarium: A Platform for In Situ Transmission Electron Microscopy in Liquid Media. Journal of Microelectromechanical Systems, 2010. 19(4): p. 885-894.

54. Nielsen, M.H., et al., Structural evolution, formation pathways and energetic controls during template-directed nucleation of CaCO3. Faraday Discussions, 2012. 159: p. 105-121.

55. Patterson, J.P., et al., Observing the Growth of Metal-Organic Frameworks by in Situ Liquid Cell Transmission Electron Microscopy. Journal of the American Chemical Society, 2015. 137(23): p. 7322-7328.

56. Schneider, N.M., et al., Electron-Water Interactions and Implications for Liquid Cell Electron Microscopy. Journal of Physical Chemistry C, 2014. 118(38): p. 2237322382.

57. Gibbs, J.W. and A.W. Smith, On the equilibrium of heterogeneous substances, in Transactions of the Connecticut Academy of Arts and Sciences, Volume III, Chapter V. and Chapter IX. 1874, Tuttle, Morehouse \& Taylor. New Haven: New Haven. p. 108248, 343-524.

58. Kashchiev, D., Nucleation: Basic theory with applications. 2000, Oxford; Boston: Butterworth Heinemann.

59. Kashchiev, D., Thermodynamically consistent description of the work to form a nucleus of any size. Journal of Chemical Physics, 2003. 118(4): p. 1837-1851.

60. Nielsen, M.H., et al., Investigating processes of nanocrystal formation and transformation via liquid cell TEM. Microscopy and Microanalysis, 2014. 20(2): p. 425-436.

61. De Yoreo, J.J., et al., Crystallization by particle attachment in synthetic, biogenic, and geologic environments. Science, 2015. 349(6247): p. 498-+.

62. Navrotsky, A., Energetic clues to pathways to biomineralization: Precursors, clusters, and nanoparticles. Proceedings of the National Academy of Sciences of the United States of America, 2004. 101(33): p. 12096-12101.

63. Ostwald, W., Studien uber die Bildung und Umwandlung fester Korper. Z. Phys. Chem., 1897. 22: p. 289.

64. Lee, J.R.I., et al., Structural development of mercaptophenol self-assembled monolayers and the overlying mineral phase during templated $\mathrm{CaCO}_{3}$ crystallization from a transient amorphous film. Journal of the American Chemical Society, 2007. 129(34): p. $10370-10381$.

65. Andreassen, J.P., R. Beck, and M. Nergaard, Biomimetic type morphologies of calcium carbonate grown in absence of additives. Faraday Discussions, 2012. 159: p. 247261.

66. Chernov, A.A. and E.I. Givargizov, Modern crystallography III : Crystal growth. Springer series in solid-state sciences. Vol. 36. 1984, Berlin: Springer.

67. Nudelman, F., et al., Spiers memorial lecture: Lessons from biomineralization: comparing the growth strategies of mollusc shell prismatic and nacreous layers in Atrina rigida. Faraday Discussions, 2007. 136: p. 9-25.

68. Young, J.R., et al., Coccolith ultrastructure and biomineralisation. Journal of Structural Biology, 1999. 126(3): p. 195-215. 
69. Marsh, M.E., POLYANION-MEDIATED MINERALIZATION - ASSEMBLY AND REORGANIZATION OF ACIDIC POLYSACCHARIDES IN THE GOLGI SYSTEM OF A COCCOLITHOPHORID ALGA DURING MINERAL DEPOSITION. Protoplasma, 1994. 177(3-4): p. 108-122.

70. Addadi, L., et al., A Chemical-Model for the Cooperation of Sulfates and Carboxylates in Calcite Crystal Nucleation - Relevance to Biomineralization. Proceedings of the National Academy of Sciences of the United States of America, 1987. 84(9): p. 27322736.

71. De Yoreo, J.J. and P.G. Vekilov, Principles of crystal nucleation and growth, in Biomineralization, P.M. Dove, J.J. DeYoreo, and S. Weiner, Editors. 2003, Mineralogical Soc America: Washington. p. 57-93.

72. Hamm, L.M., et al., Reconciling disparate views of template-directed nucleation through measurement of calcite nucleation kinetics and binding energies. Proceedings of the National Academy of Sciences of the United States of America, 2014. 111(4): p. 1304-1309.

73. Israelachvili, J.N., Intermolecular and Surface Forces. 3rd ed. 2011, Waltham, MA: Academic Press.

74. Quigley, D., et al., Sampling the structure of calcium carbonate nanoparticles with metadynamics. Journal of Chemical Physics, 2011. 134(4).

75. Raiteri, P. and J.D. Gale, Water Is the Key to Nonclassical Nucleation of Amorphous Calcium Carbonate. Journal of the American Chemical Society, 2010. 132(49): p. 17623-17634.

76. Voorhees, P.W., The theory of Ostwald ripening. Journal of Statistical Physics, 1985. 38(1-2): p. 231-252.

77. Joesten, R.L., Kinetics of Coarsening and Diffusion-Controlled Mineral Growth. Contact Metamorphism, 1991. 26: p. 507-582.

78. Zhang, H.Z. and J.F. Banfield, Interatomic Coulombic interactions as the driving force for oriented attachment. Crystengcomm, 2014. 16(8): p. 1568-1578.

79. Burton, W.K., N. Cabrera, and F.C. Frank, The growth of crystals and the equilibrium structure of their surfaces. Philosophical Transactions of the Royal Society of London Series a-Mathematical and Physical Sciences, 1951. 243(866): p. 299-358.

80. Dey, A., et al., The role of prenucleation clusters in surface-induced calcium phosphate crystallization. Nature Materials, 2010. 9(12): p. 1010-1014.

81. Gebauer, D. and H. Colfen, Prenucleation clusters and non-classical nucleation. Nano Today, 2011. 6(6): p. 564-584.

82. Gebauer, D., A. Volkel, and H. Colfen, Stable prenucleation calcium carbonate clusters. Science, 2008. 322(5909): p. 1819-1822.

83. Habraken, W.J.E.M., et al., Ion-association complexes unite classical and non-classical theories for the biomimetic nucleation of calcium phosphate. Nature Communications, 2013. 4.

84. Pouget, E.M., et al., The Initial Stages of Template-Controlled CaCO3 Formation Revealed by Cryo-TEM. Science, 2009. 323(5920): p. 1455-1458.

85. Gebauer, D., et al., Pre-nucleation clusters as solute precursors in crystallisation. Chemical Society Reviews, 2014. 43(7): p. 2348-2371.

86. Bewernitz, M.A., et al., A metastable liquid precursor phase of calcium carbonate and its interactions with polyaspartate. Faraday Discussions, 2012. 159: p. 291-312. 
87. Wallace, A.F., et al., Microscopic evidence for liquid-liquid separation in supersaturated $\mathrm{CaCO}_{3}$ solutions. Science, 2013. 341(6148): p. 885-889.

88. Wolf, S.E., et al., Early homogenous amorphous precursor stages of calcium carbonate and subsequent crystal growth in levitated droplets. Journal of the American Chemical Society, 2008. 130(37): p. 12342-12347.

89. Killian, C.E., et al., Mechanism of Calcite Co-Orientation in the Sea Urchin Tooth. Journal of the American Chemical Society, 2009. 131(51): p. 18404-18409.

90. Politi, Y., et al., Sea urchin spine calcite forms via a transient amorphous calcium carbonate phase. Science, 2004. 306(5699): p. 1161-1164.

91. DeVol, R.T., et al., Nanoscale Transforming Mineral Phases in Fresh Nacre. Journal of the American Chemical Society, 2015. 137(41): p. 13325-13333.

92. Baumgartner, J., et al., Nucleation and growth of magnetite from solution. Nature Materials, 2013. 12(4): p. 310-314.

93. Cölfen, H. and M. Antonietti, Mesocrystals: Inorganic superstructures made by highly parallel crystallization and controlled alignment. Angewandte Chemie International Edition, 2005. 44(35): p. 5576-5591.

94. Cölfen, H. and M. Antonietti, Mesocrystals and nonclassical crystallization. 2008, Chichester, England; Hoboken, NJ: Wiley.

95. Banfield, J.F., et al., Aggregation-based crystal growth and microstructure development in natural iron oxyhydroxide biomineralization products. Science, 2000. 289(5480): p. 751-754.

96. Penn, R.L., et al., Iron oxide coatings on sand grains from the Atlantic coastal plain: High-resolution transmission electron microscopy characterization. Geology, 2001. 29(9): p. 843-846.

97. Boneschanscher, M.P., et al., Long-range orientation and atomic attachment of nanocrystals in 2D honeycomb superlattices. Science, 2014. 344(6190): p. 13771380.

98. Cho, K.S., et al., Designing PbSe nanowires and nanorings through oriented attachment of nanoparticles. Journal of the American Chemical Society, 2005. 127(19): p. 7140-7147.

99. Penn, R.L., Kinetics of oriented aggregation. Journal of Physical Chemistry B, 2004. 108(34): p. 12707-12712.

100. Penn, R.L. and J.F. Banfield, Imperfect oriented attachment: Dislocation generation in defect-free nanocrystals. Science, 1998. 281(5379): p. 969-971.

101. Penn, R.L. and J.F. Banfield, Oriented attachment and growth, twinning, polytypism, and formation of metastable phases: Insights from nanocrystalline TiO2. American Mineralogist, 1998. 83(9-10): p. 1077-1082.

102. Yuwono, V.M., et al., Oriented aggregation: Formation and transformation of mesocrystal intermediates revealed. Journal of the American Chemical Society, 2010. 132(7): p. 2163-+.

103. Zhang, H.Z., J.J. De Yoreo, and J.F. Banfield, $A$ unified description of attachment-based crystal growth. Acs Nano, 2014. 8(7): p. 6526-6530.

104. Van Driessche, A.E.S., et al., The role and implications of bassanite as a stable precursor phase to gypsum precipitation. Science, 2012. 336(6077): p. 69-72. 
105. Frandsen, C., et al., Aggregation-induced growth and transformation of $b$-FeOOH nanorods to micron-sized $a-\mathrm{Fe}_{2} \mathrm{O}_{3}$ spindles. Crystengcomm, 2014. 16(8): p. 14511458.

106. Xu, A.W., et al., Polymer-mediated mineralization and self-similar mesoscaleorganized calcium carbonate with unusual superstructures. Advanced Materials, 2008. 20(7): p. 1333-+.

107. Mann, S., Biomineralization: principles and concepts in bioinorganic materials chemistry. 2001, Oxford, UK: Oxford University Press.

108. Hackney, S.A. and F.S. Biancaniello, Directional Invariance of Grain-Boundary Migration in the Pb-Sn Cellular-Transformation and the Tu-Turnbull Hysteresis. Scripta Metallurgica, 1986. 20(10): p. 1417-1422.

109. De Yoreo, J.J.N., M.H. Li, D., Smeets, P.J.M. Sommerdijk, N.A.J.M. Nucleation and particle mediated growth in mineral systems investigated by liquid-phase TEM. in Second Conference on In situ and Correlative Electron Microscopy. 2015. Saarbrucken.

\section{Figure Captions}

Figure 1-Generic sealed cell and flow cell variants of liquid phase TEM. (a) Side-view schematic of hermetically sealed liquid cell. Silicon nitride membranes suspended from silicon wafers separated by a spacer layer enclose the liquid layer imaged in the TEM. Optional features such as electrodes in the imaging area may provide additional functionality. (b) Assembled liquid cell on a custom TEM stage. Wires leading from the cell to the internal section of the stage connect the liquid cell to external electronics. (c) Dual-inlet flow stage and side view of sample region (inset). Syringes pump reagent solutions through two separate inlets. Liquid streams combine into a single solution stream shortly before flowing through and around the liquid cell, and exit the stage via an outlet tube connected to a waste container. (Inset) Liquid cell assembled from two silicon wafers supporting nitride membranes, separated by a spacer layer, as positioned in TEM stage with o-rings providing the barrier between liquid and vacuum environments. Panels (a and b) are adapted from Ref. (Nielsen et al. 2012) and reproduced with permission by The Royal Society of Chemistry. Panel (c) is reproduced from Ref. (Nielsen et al. 2014b) and used with permission by AAAS.

Figure 2 - Resolution of different forms of electron microscopy in liquid. Theoretical maximal resolution versus water thickness for TEM, STEM and SEM. The resolution was calculated for typical TEM and STEM instrument parameters at $200 \mathrm{keV}$ beam energy, and for the imaging of Au nanoparticles at the bottom of a layer of water for TEM, and at the top of the layer for STEM. 
The resolution obtained in SEM just below the liquid-enclosing membrane does not depend on the liquid thickness (see text). Experimental data points are shown for Au nanoparticles in TEM, STEM and SEM with a 30-nm-thick SiN window, and for PbS nanoparticles in water imaged with STEM35. The error bars represent experimental errors. From Ref. [15] and reproduced with permission by Macmillan Publishers Limited.

Figure 3 - Nucleation of Au nanoparticles. (a-d) Sequence of liquid cell TEM images showing the nucleation of Au nanoparticles from a gold chloride solution containing citrate as a capping agent. As seen in the example highlighted by the circle in (e-n), which were taken from the region delineated by the rectangular box in (a), many of the nascent nuclei fail to reach the point of spontaneous growth and instead fluctuate in size until they disappear. This behavior demonstrates that particle formation results from unstable fluctuations, the hallmark of classical nucleation. Times in s: (a) 0.0, (b) 34.0, (c) 68.1, (d) 103.2, (e) 2.1, (f) 8.0, (g) 16.4, (h) 34.9, (i) 36.6, (j) 44.8, (k) 60.5, (l) 73.1, (m) 93.3, and (n) 103.2. Scale bars: (a-d) $500 \mathrm{~nm}$, (e) 200nm. Scale in (f-n) is same as (e). From [60] and reproduced with permission by the Microscopy Society of America.

Figure 4 - Series of LP-TEM images showing concurrent formation of multiple $\mathrm{CaCO}_{3}$ phases. Frames show simultaneous nucleation and growth of calcite crystals (rhombohedra) and either ACC or vaterite (ellipsoidal particle). The nitride window edge is visible at the bottom right corner. From [41] and reproduced with permission by the American Association for the Advancement of Science.

Figure 5 - (A-F) Time series of LP-TEM images showing $\mathrm{CaCO}_{3}$ nucleation via a two-step process. The first phase to form is amorphous $\mathrm{CaCO}_{3}$ (ACC) $(\mathrm{a}, \mathrm{b})$. This is followed by surface nucleation of aragonite $(\mathrm{c}, \mathrm{d})$ and consumption of the ACC $(\mathrm{e}, \mathrm{f})$. Just before the moment of aragonite nucleation, the ACC partcle shrinks in size, indicating expulsion of its structural water. Scale bars: $500 \mathrm{~nm}$. From Ref. [109] and reproduced with permission by Elsevier Inc.

Figure 6 - Formation pathways of $\mathrm{CaCO}_{3}$. Amorphous phase and three anhydrous crystalline phases form directly from solution. Nucleation of vaterite and aragonite on existing amorphous 
particles leads to direct transformation from amorphous to crystal in these multi-step pathways. Crystal to crystal transformation also observed in the case of calcite forming on aragonite and growing at the expense of the initially formed phase. Scale bars $500 \mathrm{~nm}$ and $2 \mathrm{~nm}^{-1}$ for images and diffraction data, respectively. Adapted from Ref. [41].

Figure 7 - Post-nucleation growth rates. Data plotted for ACC (a), calcite (b), and vaterite (c). In all cases linear growth rates are observed, either with a single rate or with two distinct rates. Lateral off-sets $(b, c)$ have been set for clarity. Measurements from repeated experiments at the same conditions are identified by '-\#' in the panel legends. Experimental conditions for each experiment can be found in Ref. [41], from which the figure is adapted.

Figure 8 - (a) and (b) LP-TEM images of (a) PSS solution and (b) Ca-PSS solution showing globules are only observed in presence of Ca. (c) Zeta potential for the Ca-PSS solution and the solution containing only PSS (pure) as indicated (d) FTIR spectrum showing the shift in the peak associated with the symmetric stretch of the sulfonate group upon addition of $\mathrm{CaCl}$ to PSS solution. The shift from the Ca-free (pure) to the Ca-PSS, as indicated, shows that the $\mathrm{Ca}^{2+}$ ions bind to the sulfonate groups. Adapted from [10].

Figure 9 - (e) LP-TEM image from a time-lapse series exhibiting many nuclei in Ca-PSS globules and an electron diffraction pattern (inset) showing they are amorphous. Scale bar: 50 nm. Adapted from [10].

Figure 10 - (a) Time series of LP-TEM images showing ACC nucleation and growth within a globule. In absence of PSS, vaterite forms randomly (not shown). Scale bar: $20 \mathrm{~nm}$. (b) Growth rates of vaterite particles formed in the absence of PSS and ACC particles within Ca-PSS globules. Adapted from Ref. [10] by permission of Macmillan Publishers Ltd.

Figure 11 - (a to $\mathbf{g})$ Time series of LP-TEM images showing typical dynamics of OA by ferrihydrite nanoparticles. The surfaces of particles I and II made transient contact at many points and orientations (points 1-1, 1-2, 2-3 and 3-4) before finally attaching and growing 
together (points 3-5). Scale bars: $5 \mathrm{~nm}$. h: High-resolution image of interface in (g) showing twin structure (inclined twin plane). The white dashed line in $(\mathrm{g})$ presents the original boundary of the attached particle. From [8] and reproduced with permission by the American Association for the Advancement of Science.

Figure 12 - High-resolution LP-TEM images showing the two types of interfaces formed during OA of ferrihydrite nanoparticles: (a) (101) twin interface and (b) defect free interface. Panel (a) adapted from [8].

Figure 13 - (a) Relative translational and angular speeds leading up to attachment of two ferrihydrite nanoparticles. The sudden increase in speed occurs when the particles are separated by $5 \AA$ to $10 \AA$. (b) Dependence of interface growth rate on curvature derived from dependence of interface length on time after the moment of attachment for two different OA events. The maximum error from delocalization is estimated to be less than $1 \mathrm{~nm}$. From [8] and reproduced with permission by the American Association for the Advancement of Science.

Figure 14 - Times series of LP-TEM images showing formation of a defect-free interface between two ferrihydrite nanoparticles when the initial attachment event occurs with a slight misalignment. Two edge dislocations denoted in $(\mathbf{b}, \mathbf{c})$ by the dashed lines translate to the right, leaving a defect-free structure in (d). From [8] and reproduced with permission by the American Association for the Advancement of Science.

Figure 15 - Growth of ferrihydrite (iron oxyhydroxide) via oriented nanoparticle attachment. (af) Sequence of liquid cell TEM images showing a dumbbell-shaped ferryhidrite particle formed during an earlier aggregation event, attaching to a larger crystal formed from many such events. As the dumbbell-shaped particle approaches the larger crystal (a-b), it feels the attractive force that drives oriented attachment and undergoes a significant clockwise rotation (b-d) before jumping to contact (c-d). Immediately following attachment, there are a number of lattice defects in the boundary region (d-e), but these quickly translate to the surface and are expelled, causing a counter-clockwise rotation of the particle about the new interface (f). Times in s: (a) 0 , 
(b) 2.4, (c) 5.6, (d) 6.6, (e) 7.4 and (f) 32.2. Scale bar in (a) is $10 \mathrm{~nm}$ and applies to all images. From [60] and reproduced with permission by the Microscopy Society of America.

Figure 16 - Dependence of relative orientation vs. time following attachment of dumbbellshaped ferryhidrite particle shown in Fig. 15. The particle rotates counter clockwise at a rate of $87 \mathrm{mrad} \mathrm{s}^{-1}$ before reaching an equilibrium position. From [60] and reproduced with permission by the Microscopy Society of America.

Figure 17 - Growth of $\mathrm{CaCO}_{3}$ by particle aggregation. (a-f) Sequence of liquid cell TEM images showing small spherical $\mathrm{CaCO}_{3}$ particles with no apparent lattice structure interacting with and attaching to either one another or to a larger composite crystal. Times in s: (a) 0, (b) 0.8, (c) 1.3, (d) 3.3, (e) 4.5 and (f) 6.5. Scale bar in (a) is $5 \mathrm{~nm}$ and applies to all images. From [60] and reproduced with permission by the Microscopy Society of America.

Figure 18 - Ostwald ripening of ferrihydrite particles (a-f) Sequence of liquid cell TEM images showing the dissolution of a small ferrihydrite particle with positive curvature near a large ferrihydrite surface with negative curvature. Times in s: (a) 0, (b) 4.6, (c) 7.6, (d) 10.2, (e) 12.6 and (f) 14.6. Scale bar in (a) is $5 \mathrm{~nm}$ and applies to all images. From [60] and reproduced with permission by the Microscopy Society of America.

Figure 19 - (a) Decrease in diameter $D$ from initial diameter $D_{0}$ with time t plotted as $\left(D / D_{0}\right)^{3}$ vs. $t$ for an individual particles near a concave surface of a much larger particle. (Particle 1 is the same one shown in Fig. 18.) From [60] and reproduced with permission by the Microscopy Society of America. 

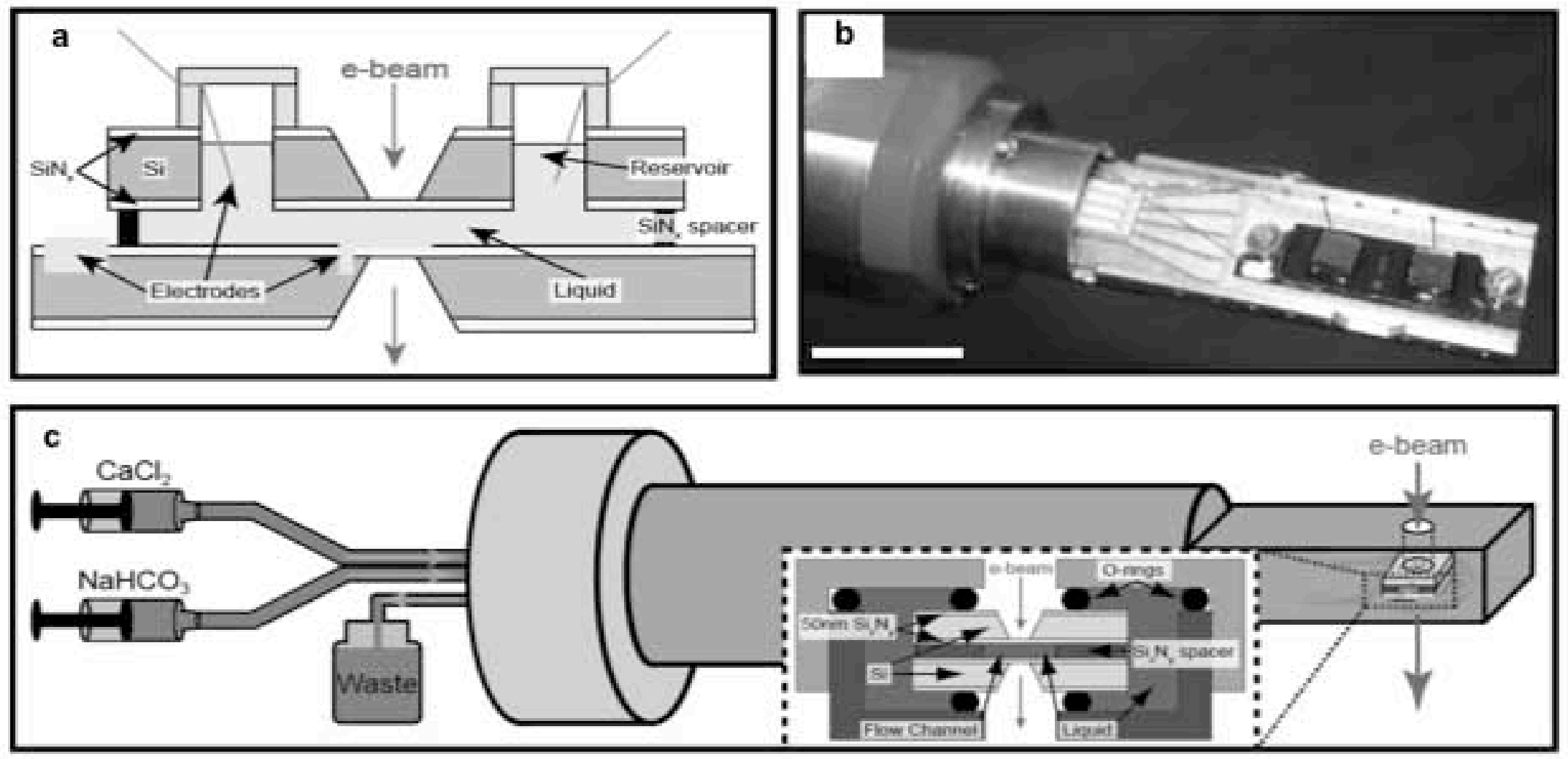

Figure 1 


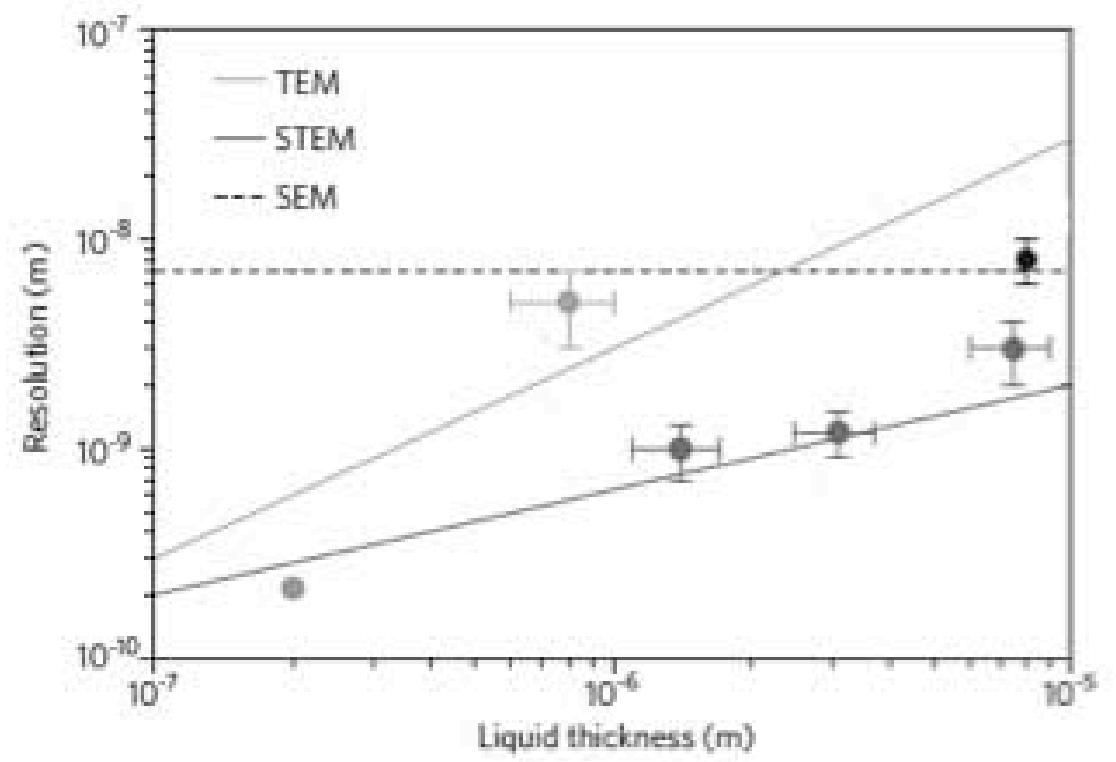

Figure 2 

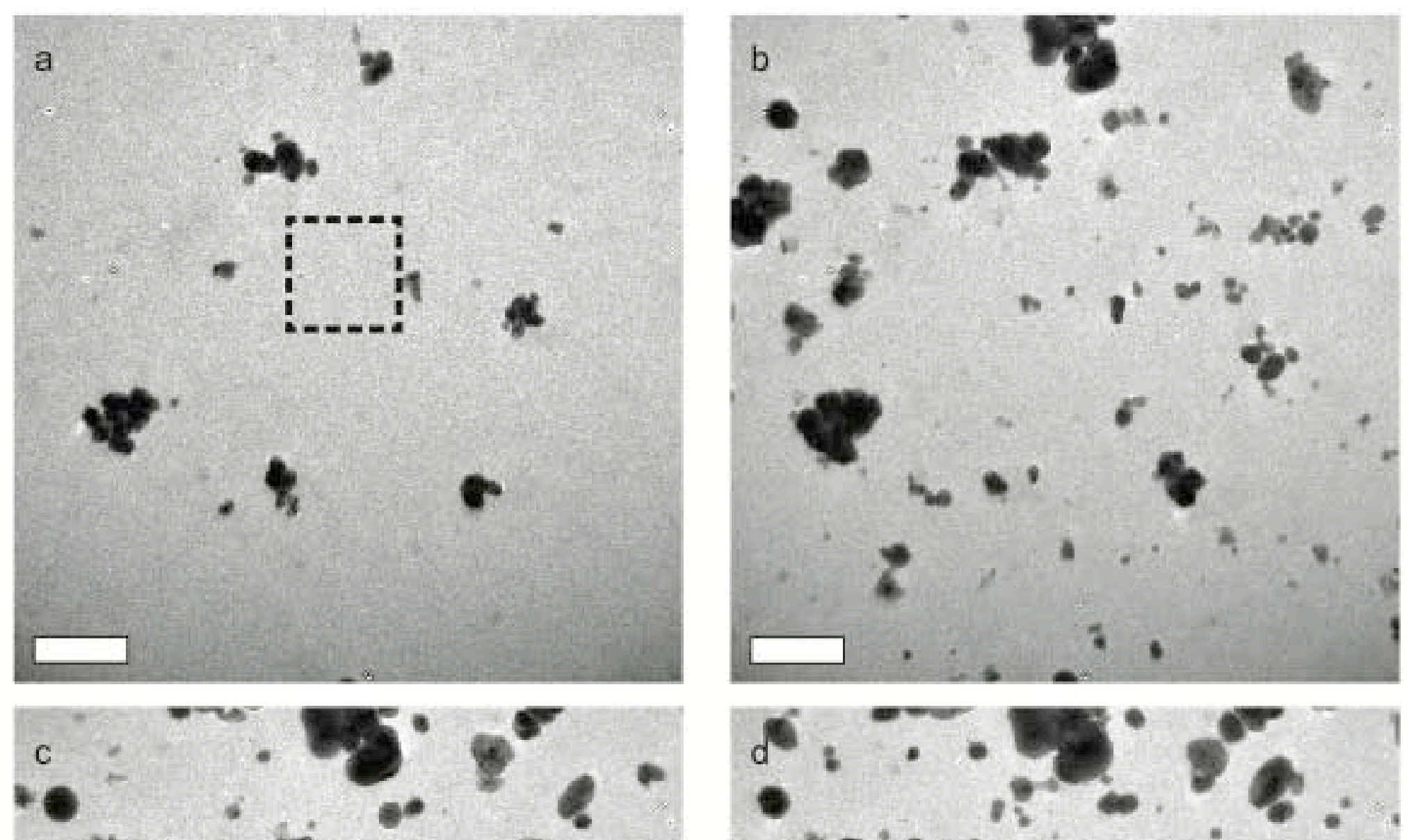

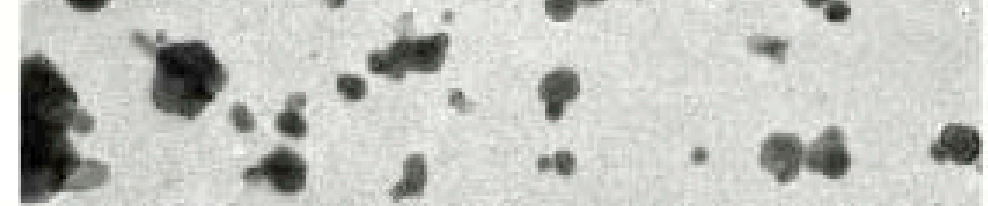

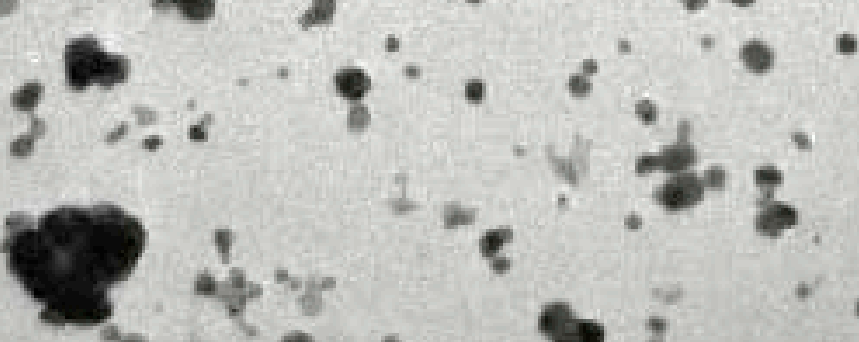

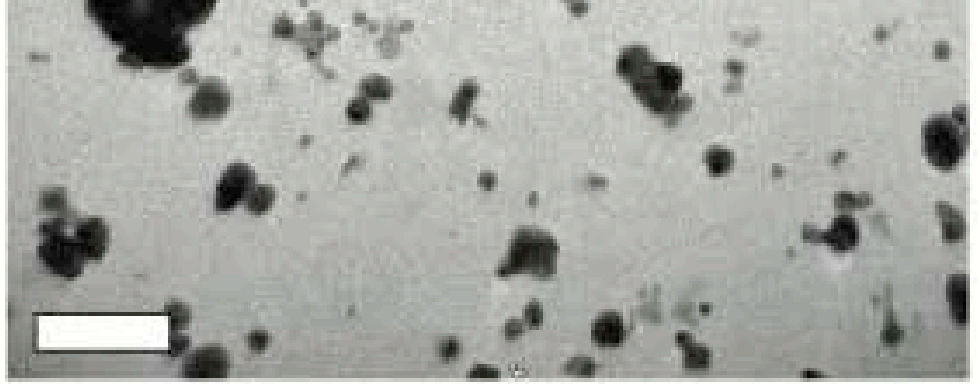

E 1
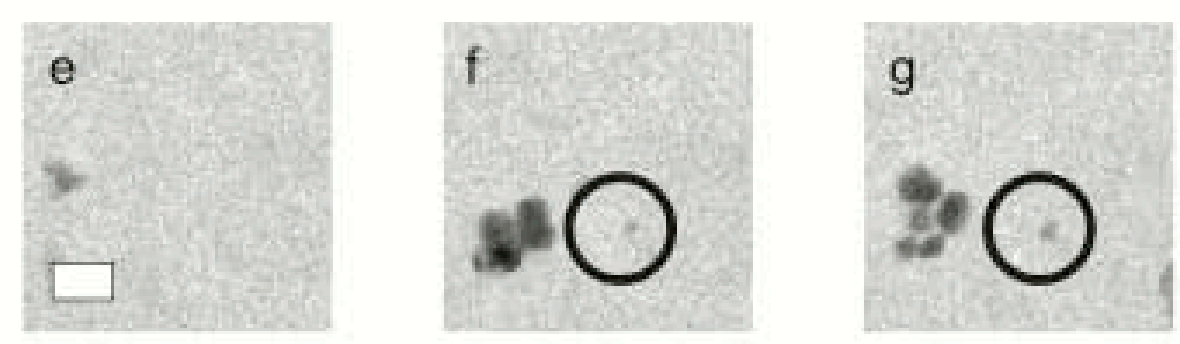

j $=$

k $\quad$ it
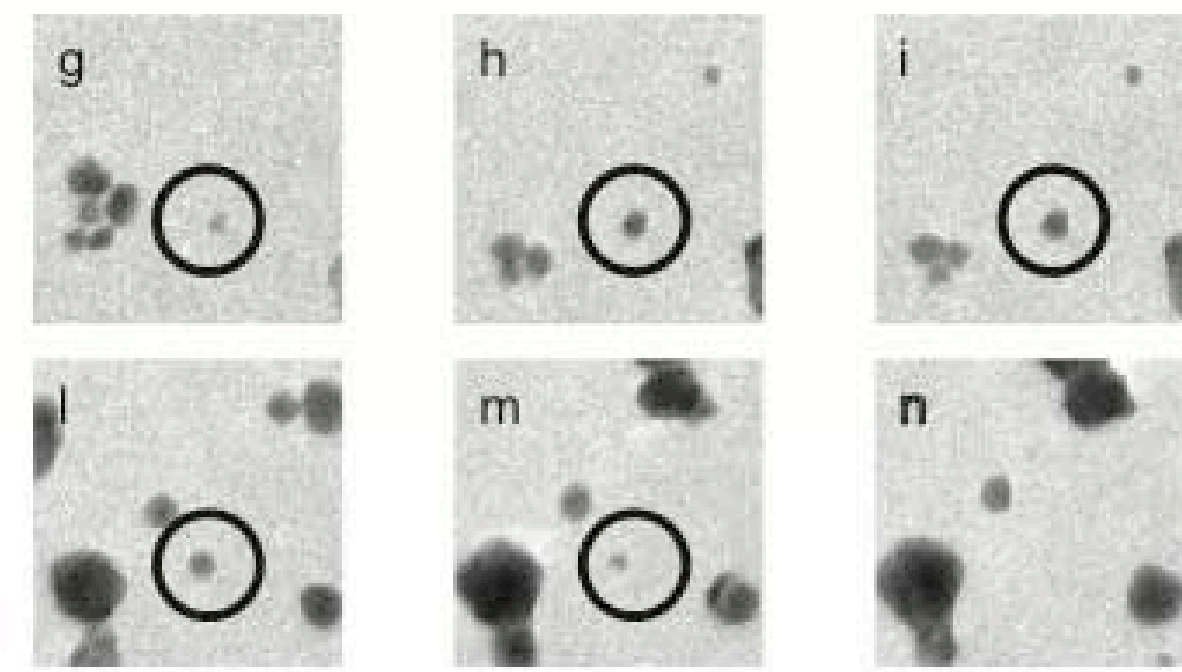

$, 0,-O_{1}$

$\odot$.
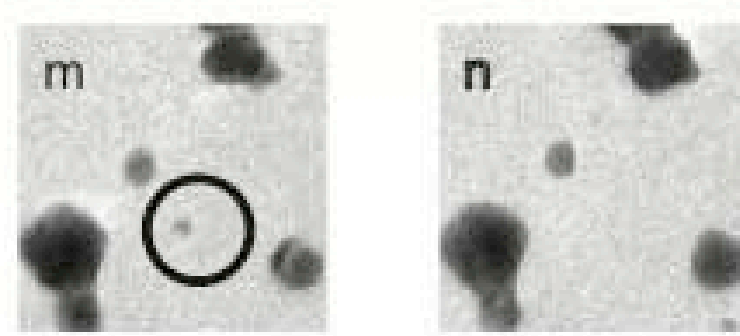

a

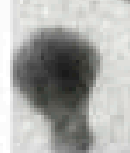

Figure 3 


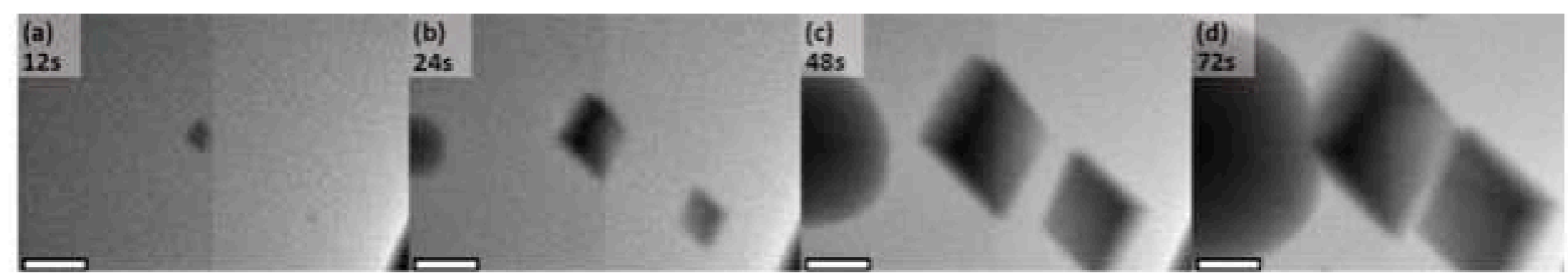

Figure 4 

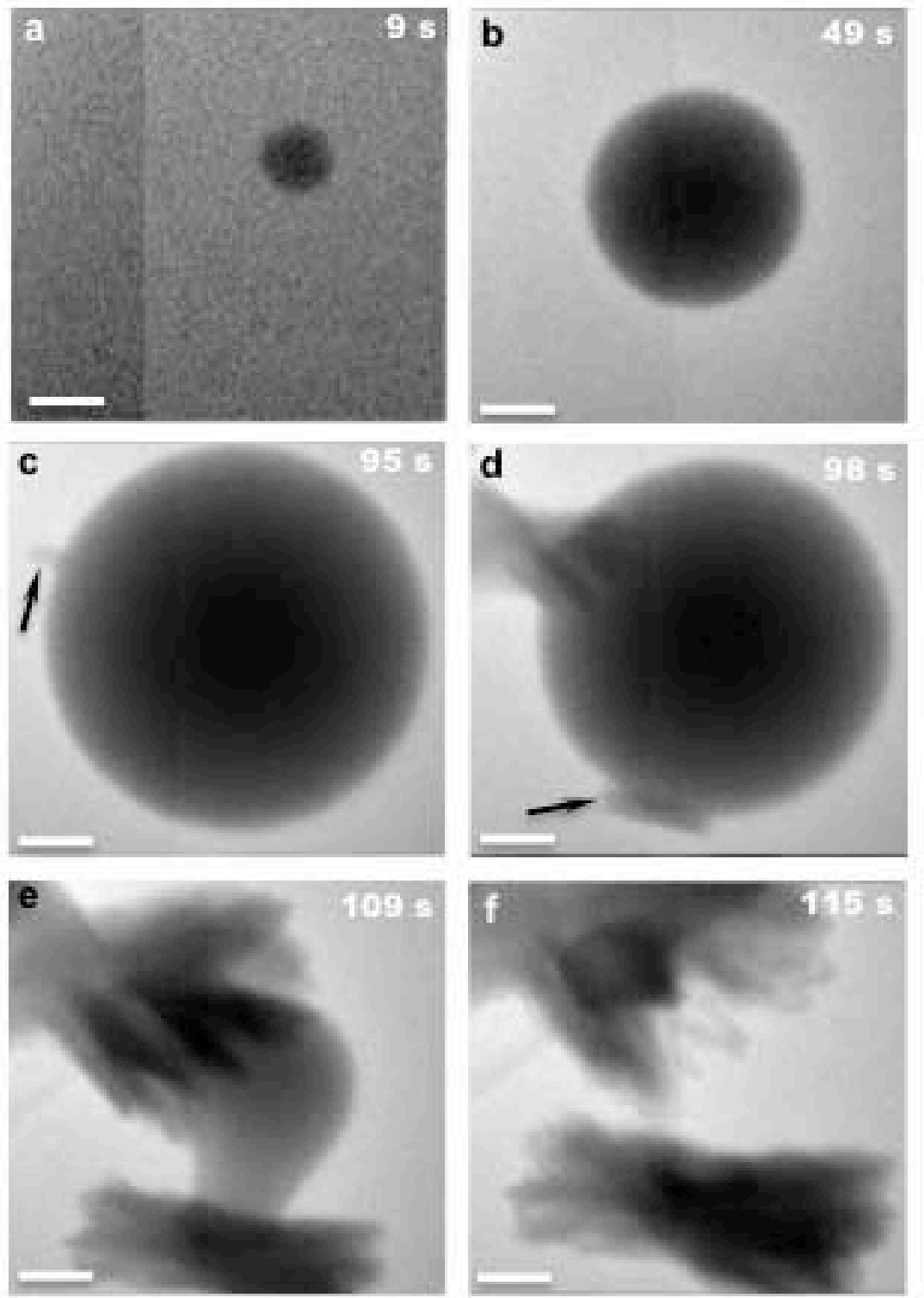

Figure 5 


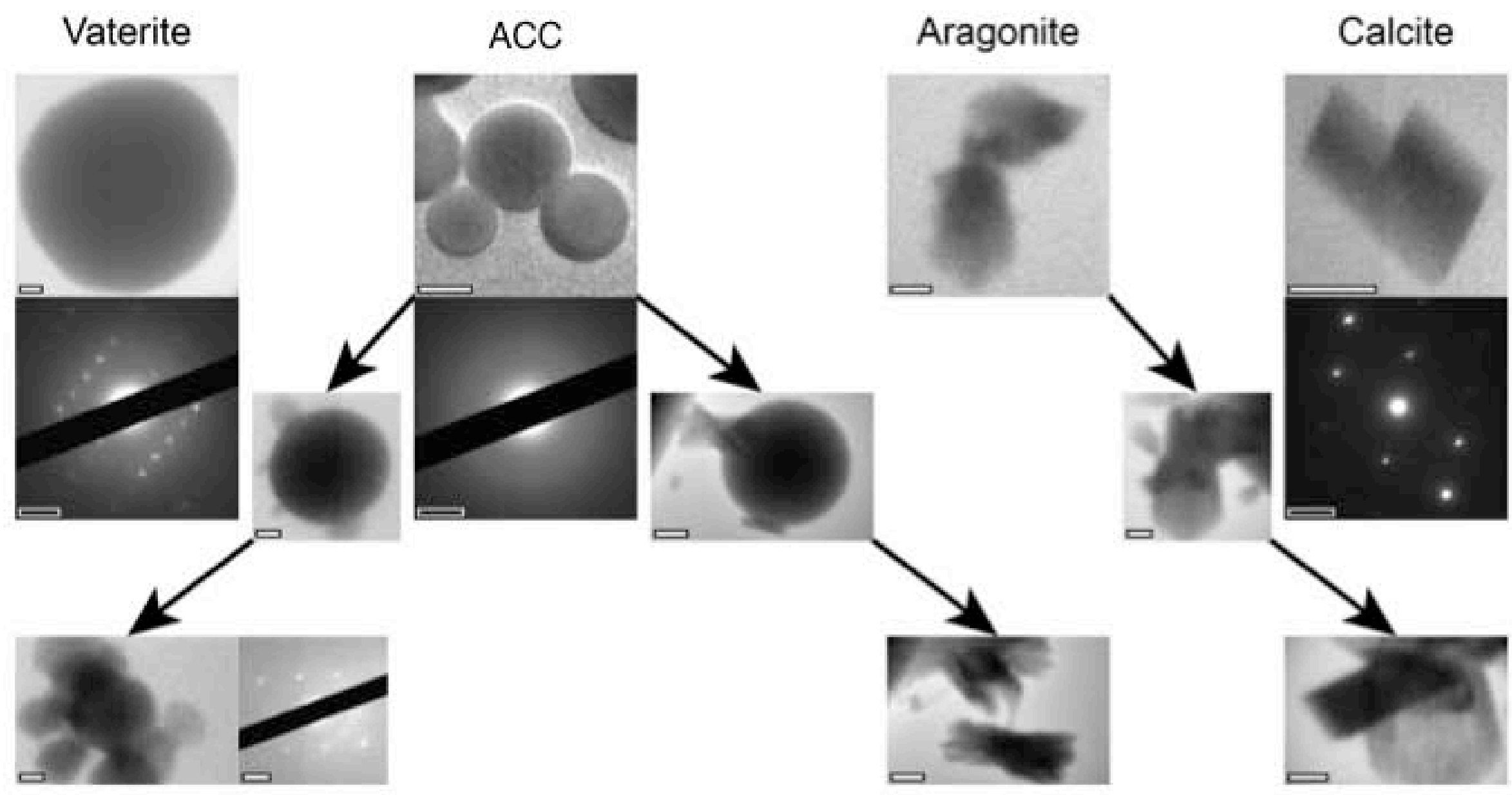

Figure 6 


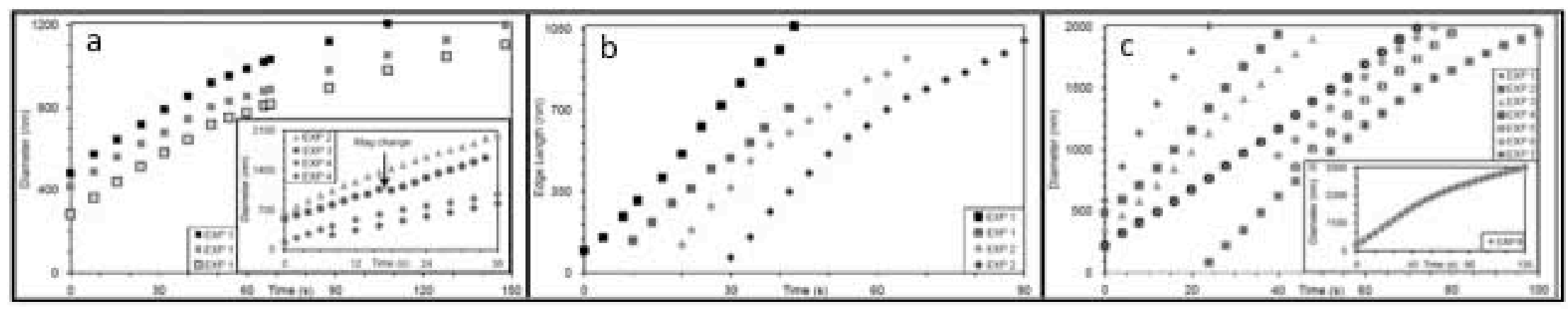

Figure 7 

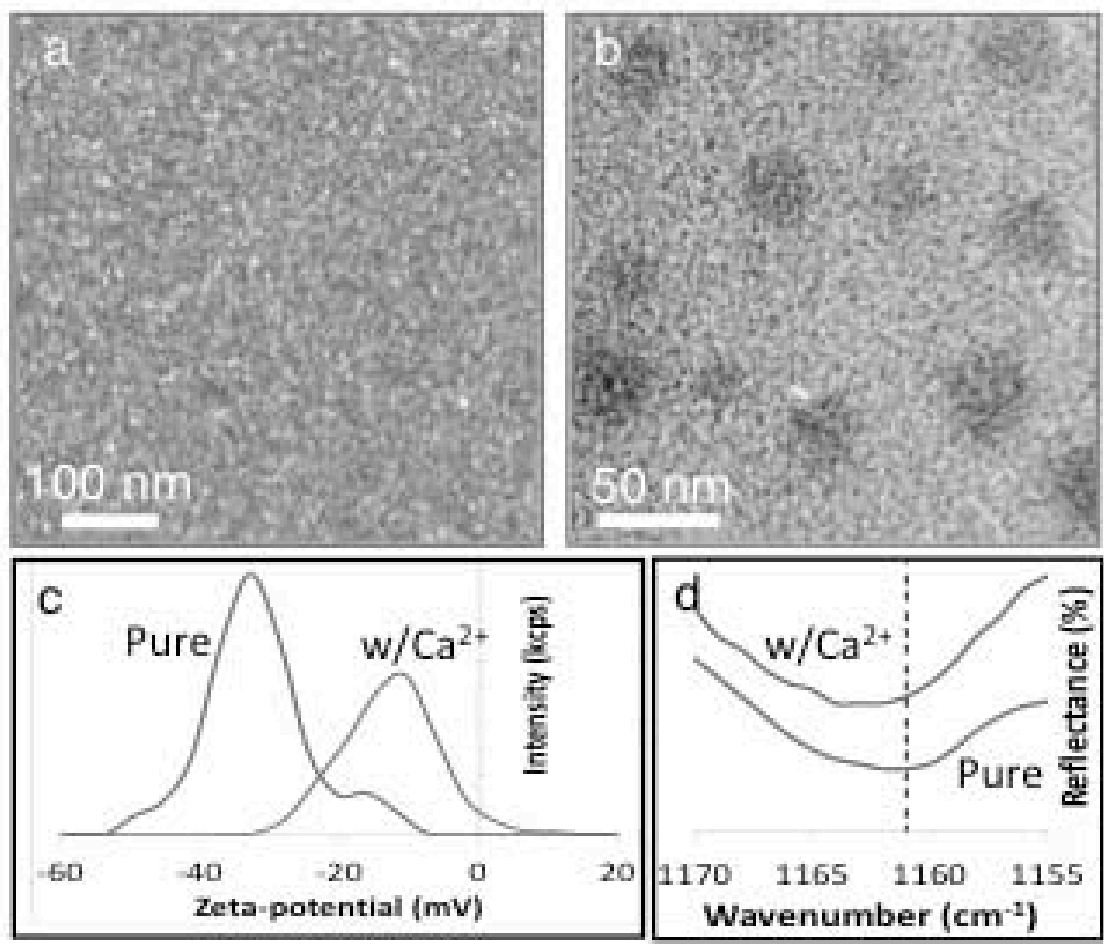

Figure 8 


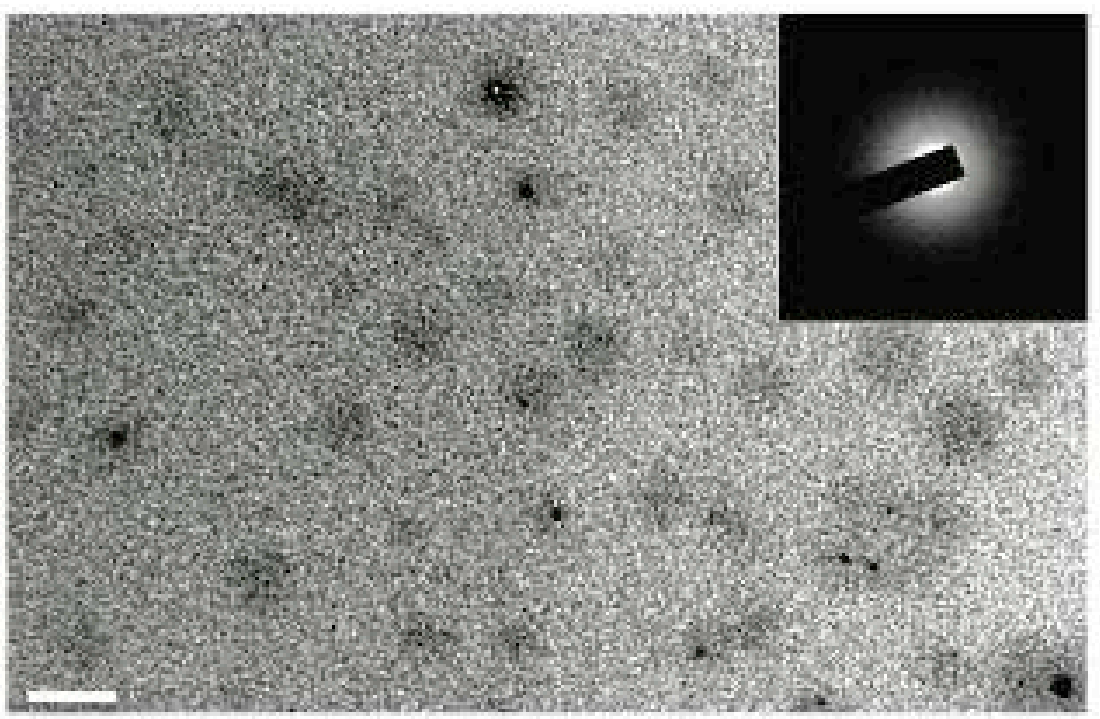

Figure 9 

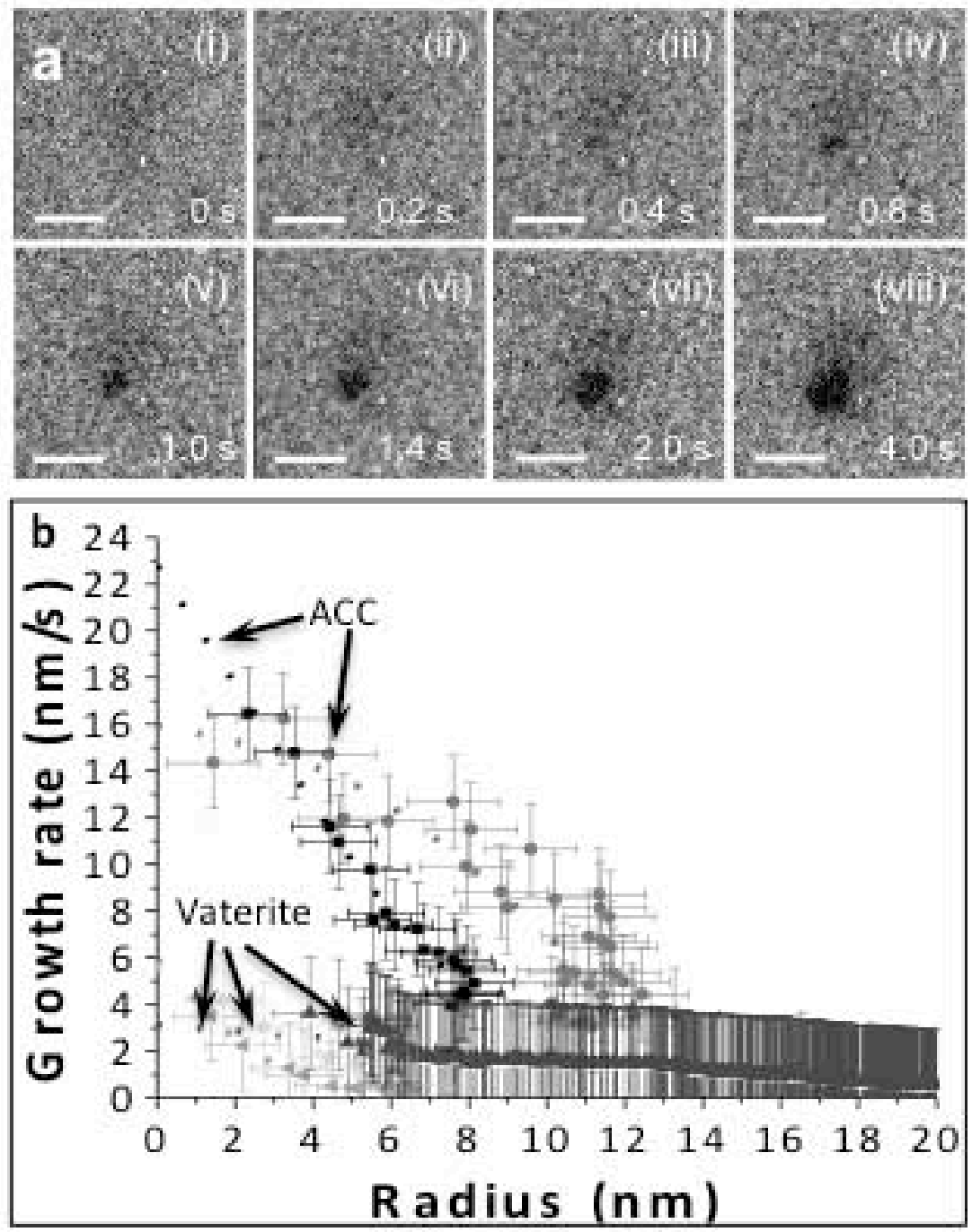

Figure 10 


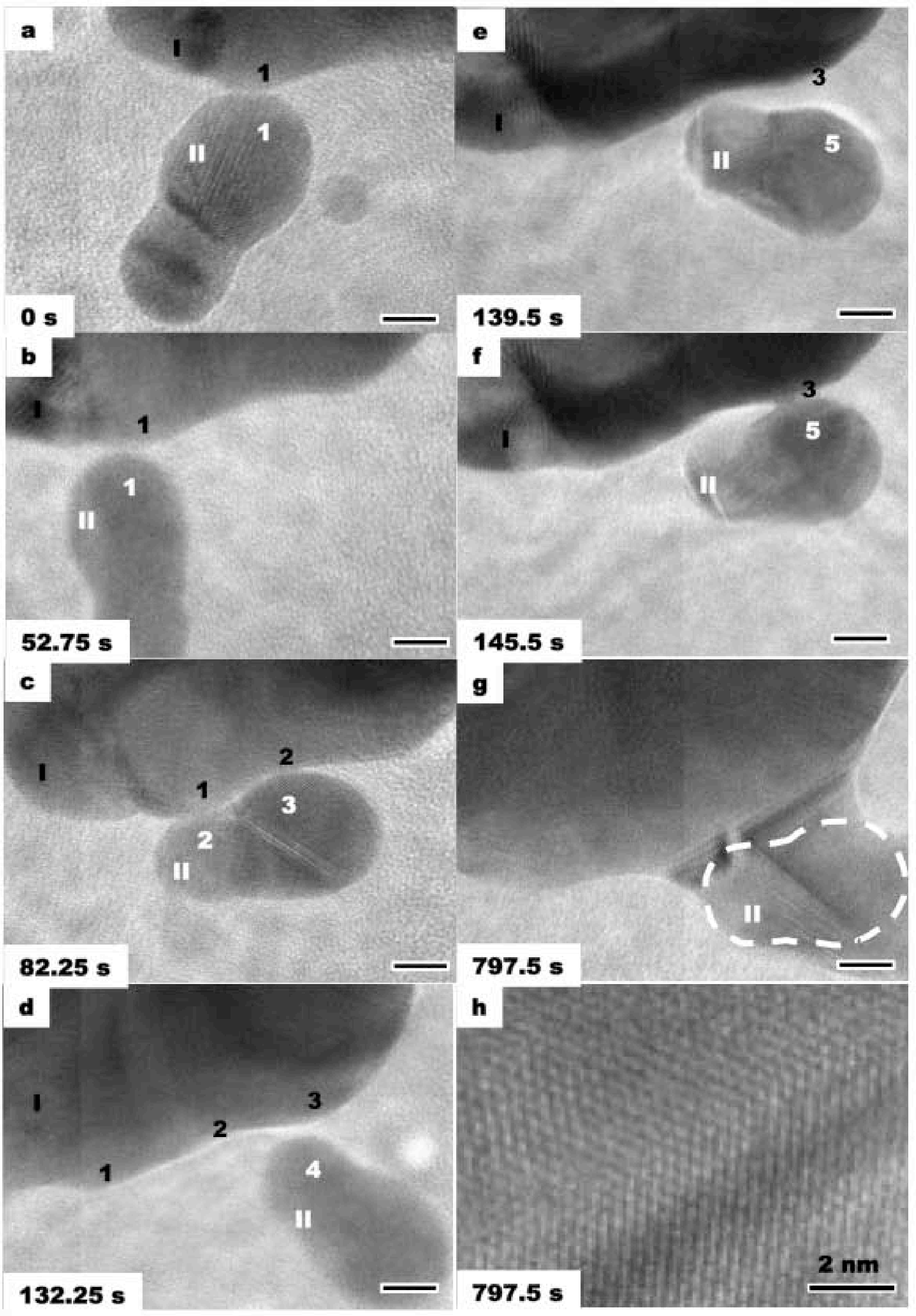

Figure 11 


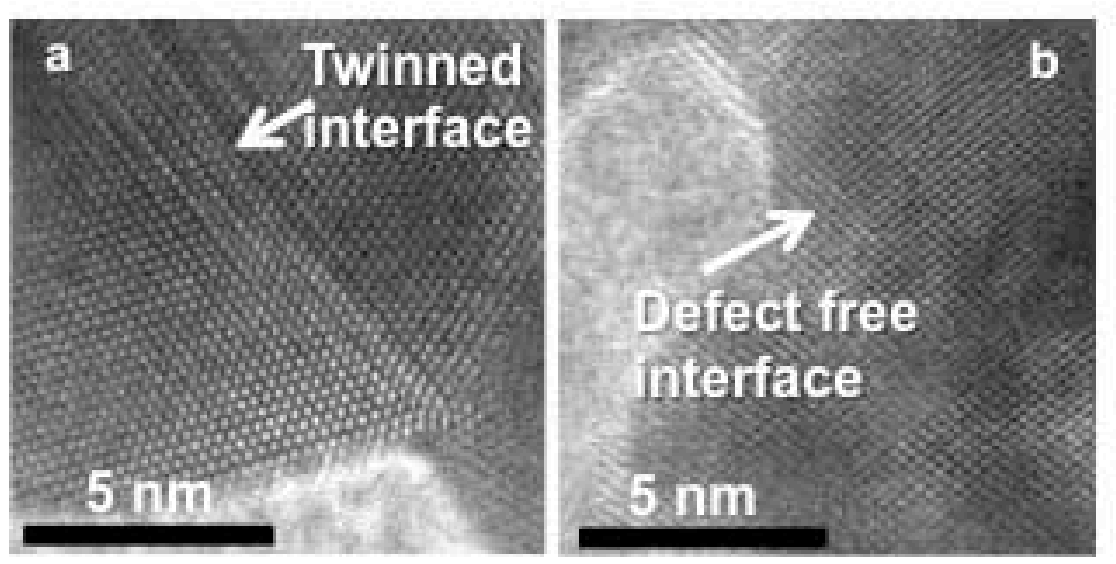

Figure 12 

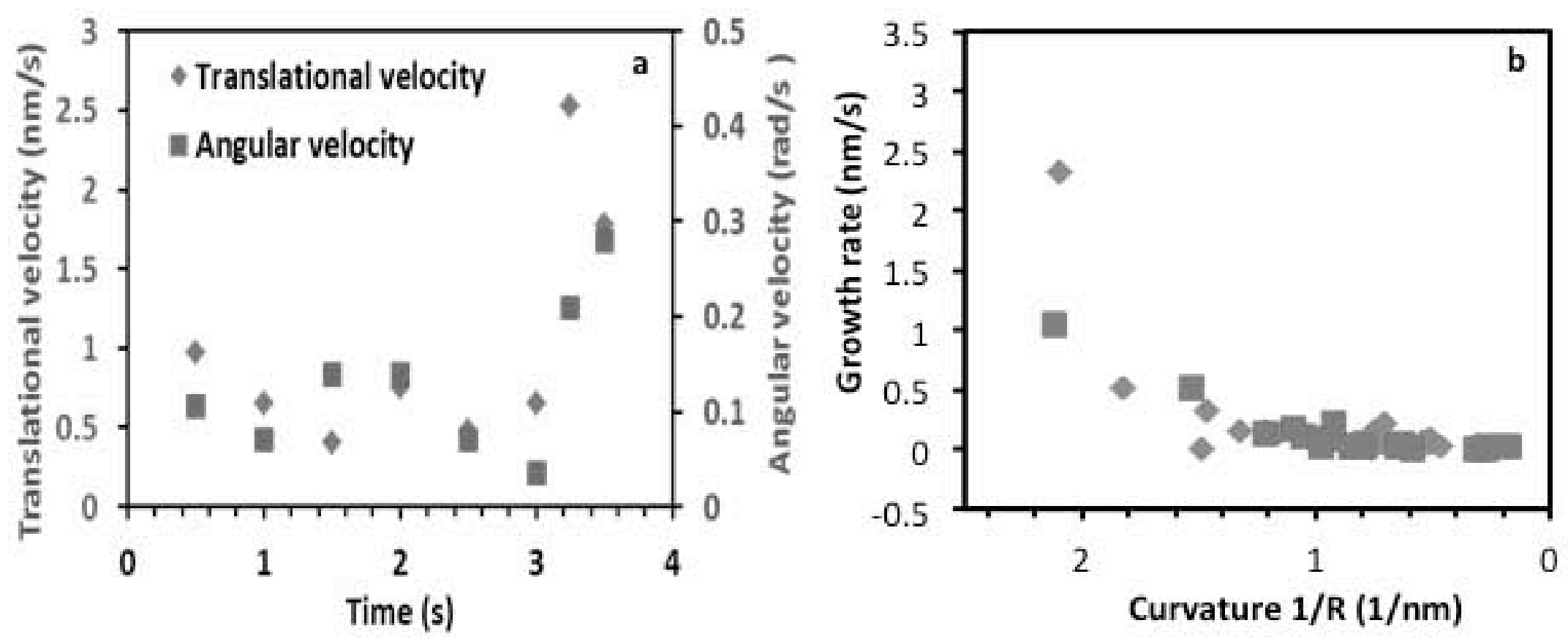

Figure 13 


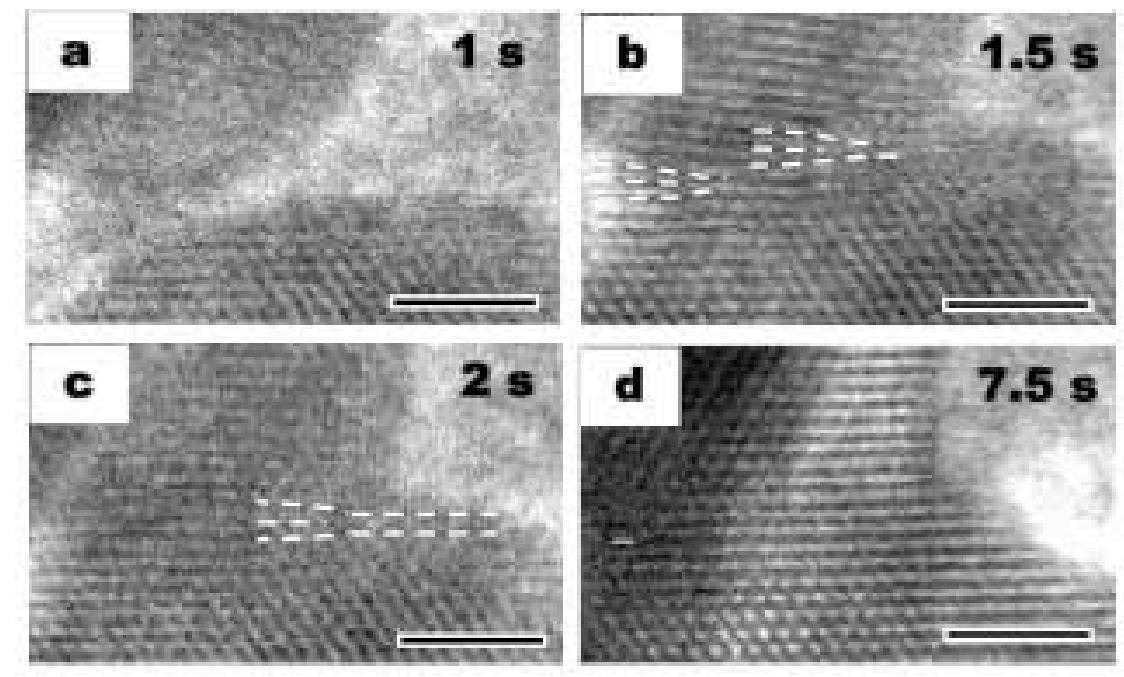

Figure 14 

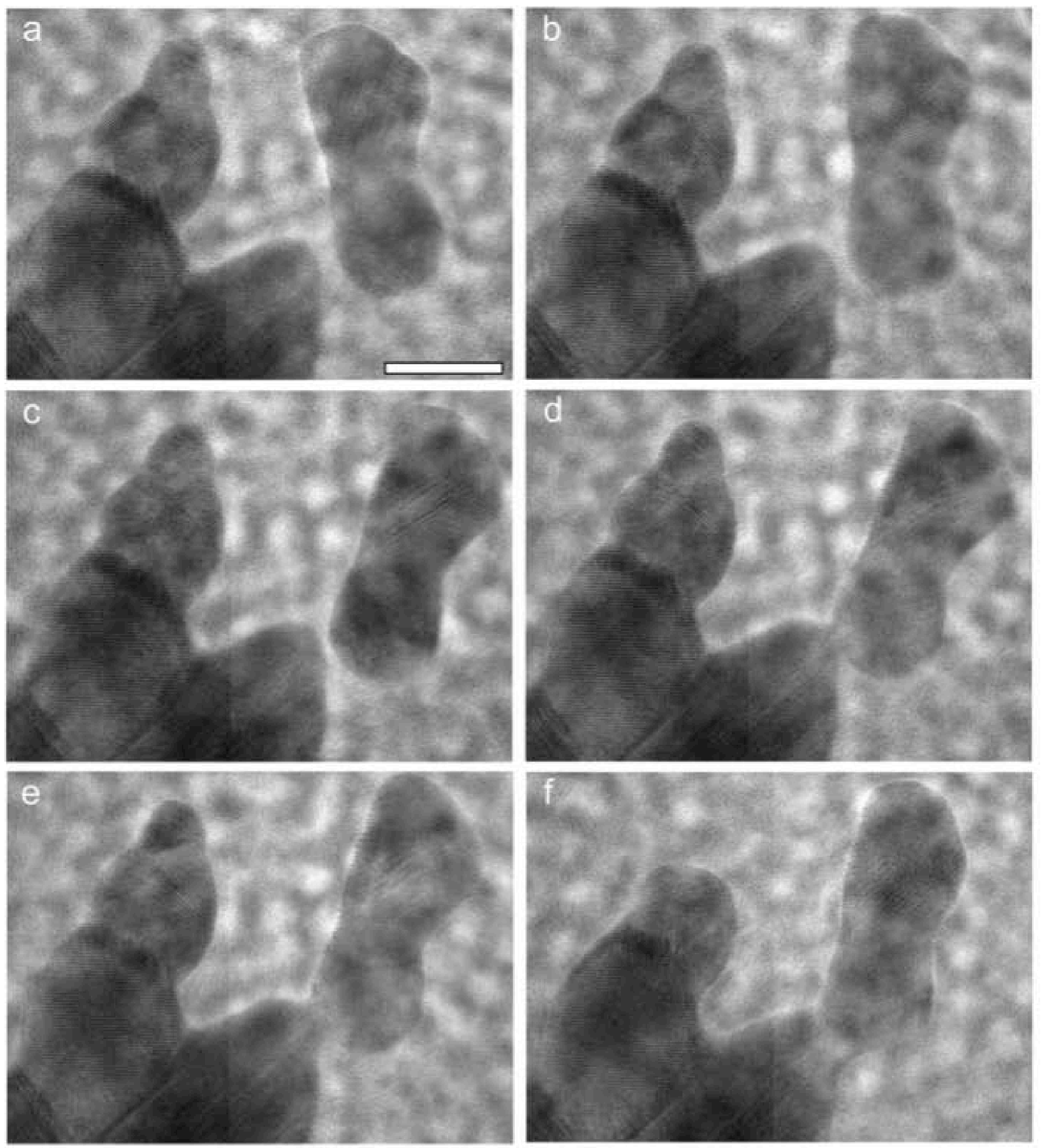

Figure 15 


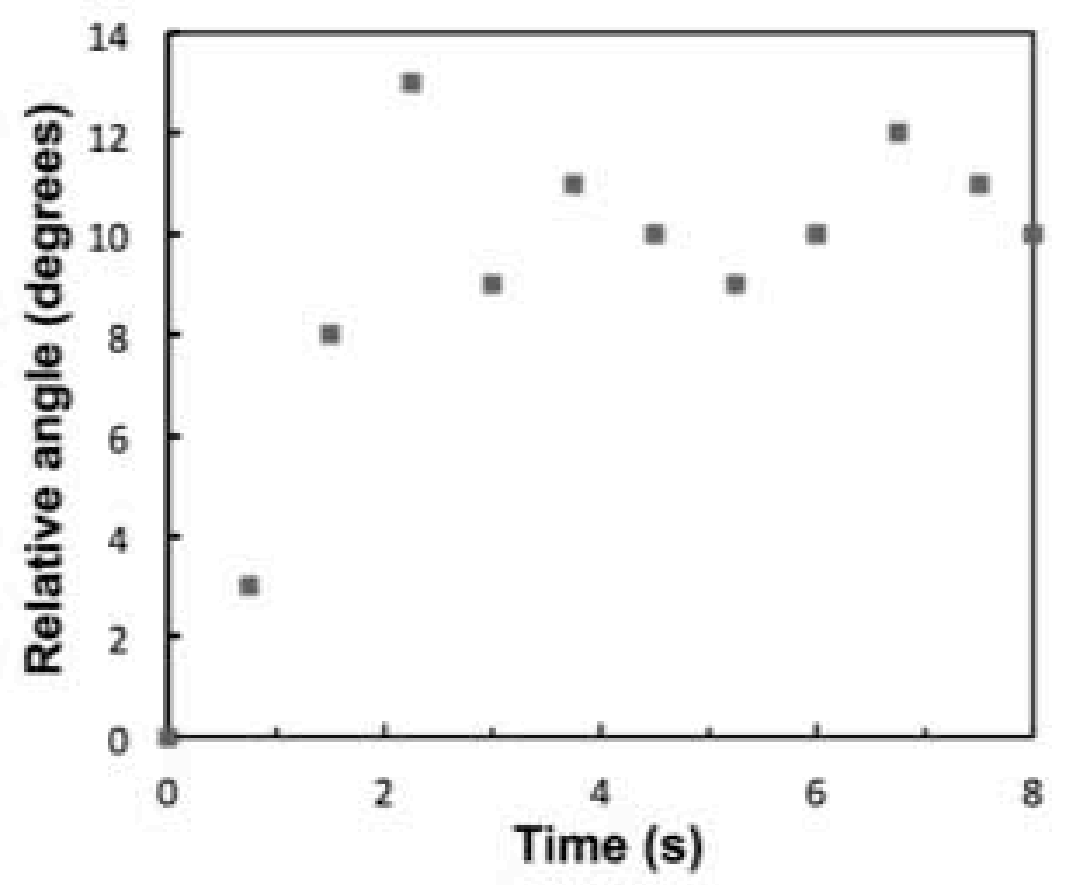

Figure 16 

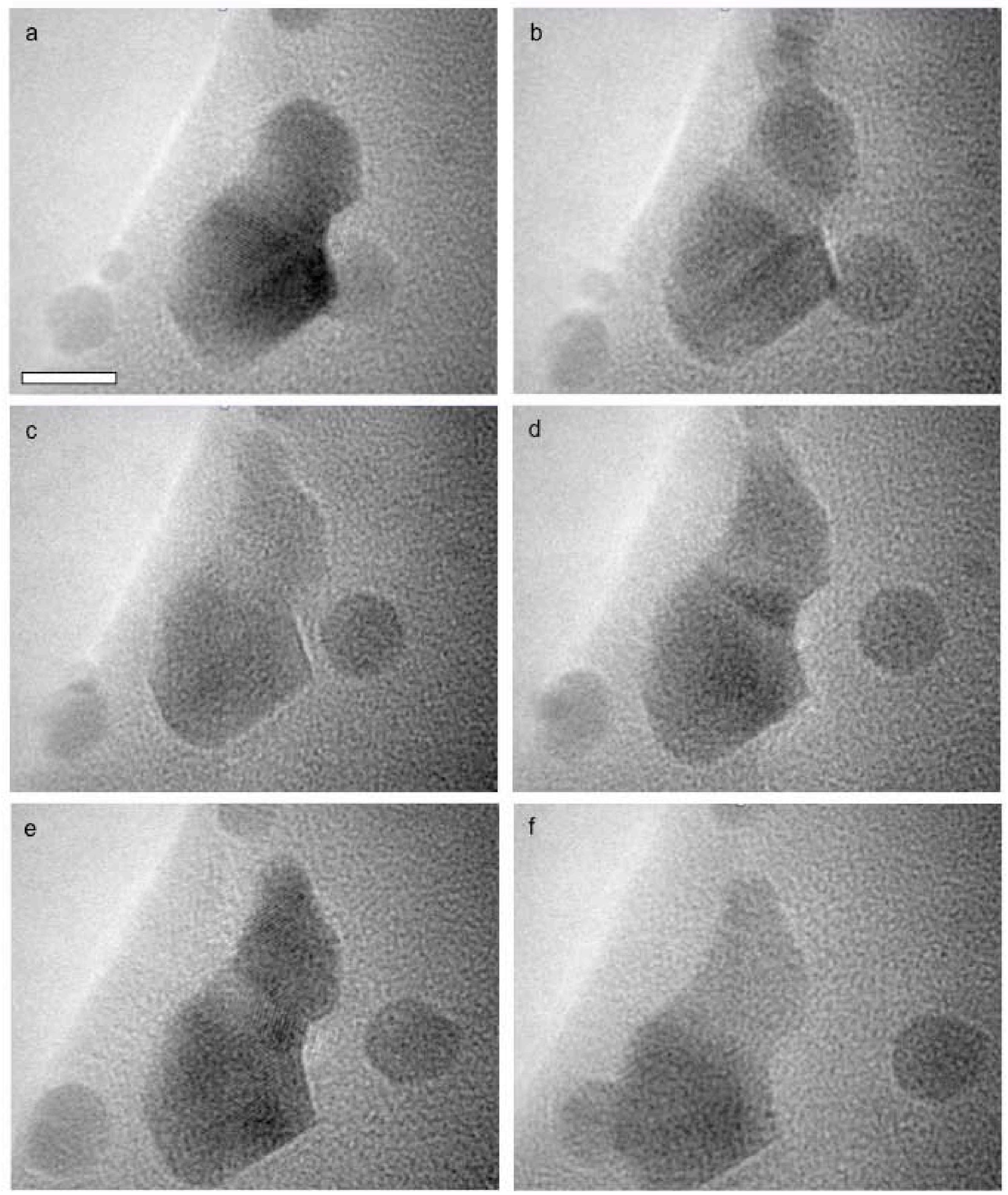

Figure 17 

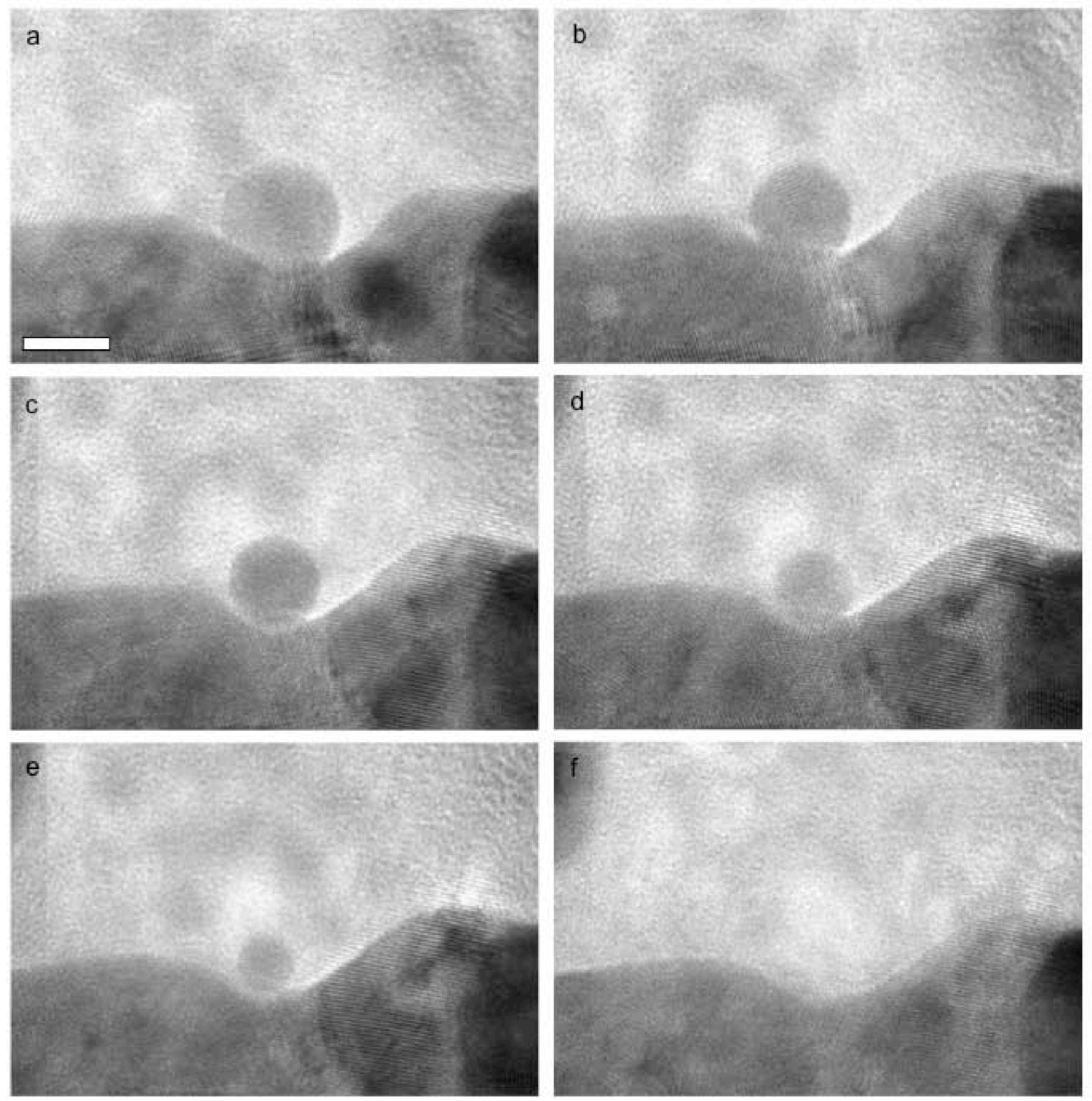

Figure 18 


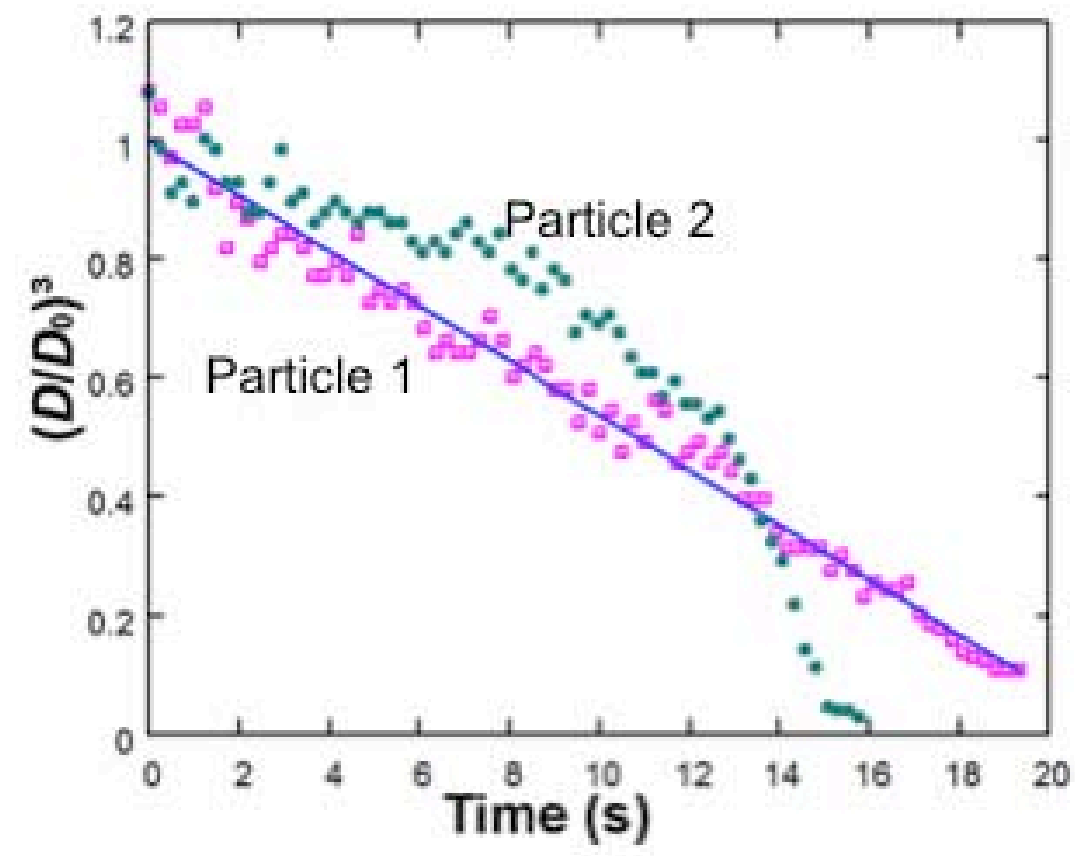

Figure 19 